\title{
Novel hybrid WDM/TDM PON architectures to manage flexibility in optical access networks
}

Bart Lannoo, Goutam Das, Abhishek Dixit, Didier Colle, Mario Pickavet, Piet Demeester

Ghent University - iMinds, Gaston Crommenlaan 8, box 201, B-9050 Gent, Belgium

Phone: +32 93314998

Fax: +3293314899

Email: bart.lannoo@intec.ugent.be

Website: http://www.ibcn.intec.ugent.be

Abstract: Different hybrid WDM/TDM PON architectures are compared in terms of flexibility, simplicity (affecting the cost), insertion loss (affecting the reach) and security. Special attention is given to the flexibility aspect in next generation optical access networks by designing different architectures with a different degree of flexibility, which are able to cope with different ranges of dynamic bandwidth allocation (DBA) possibilities. This paper assesses the degree of architectural flexibility needed to deal with some important flexibility advantages. It is shown that mostly a partially flexible architecture fulfils the needs. The architectures are then further evaluated from a cost and reach perspective. In this way, we provide a complete comparison considering all the key aspects of access network design. It is shown that a hybrid WDM/TDM PON with a partially flexible architecture in the first remote node can be an interesting candidate for next-generation optical access networks.

Keywords: optical access, PON architectures, hybrid WDM/TDM, flexibility, dynamic bandwidth allocation

\section{Introduction}

Currently, telecom operators are adapting their broadband access networks for offering highly demanding services such as high-definition TV, interactive gaming, video-conferencing, etc. Optical fiber networks are considered the most future-proof next generation access (NGA) technologies. Nowadays, the most used optical fiber access network configuration is a (power splitting) time division multiplexing (TDM) passive optical network (PON), with Ethernet PON (EPON) and gigabit-capable PON (GPON) as the two most important standards [1]-[3]. The currently deployed EPON or GPON systems, however, are unable to provide the expected residential data rates by the year 2020, being a sustainable data rate 
of $500 \mathrm{Mbps}$ per user (or subscriber) and a peak data rate of 1 Gbps per user [4]. Typically these PON systems are using a separate wavelength (of 1 or $2.5 \mathrm{Gbps}$ ) for down- and upstream, and both wavelengths are then shared between multiple users (e.g., 16, 32, 64). As the users share the same pool of capacity, competition may arise and traffic requests may not be honored due to congestion.

The mentioned capacity bottleneck for TDM PONs is currently tackled by the standardization activities for the 10G xPON systems (10G EPON and 10G GPON, respectively). The physical access bit rate is pushed to $10 \mathrm{Gbps}$ per wavelength, firstly for the downlink part and secondly in a symmetric offer for the uplink part. Another attractive PON solution is the wavelength division multiplexing (WDM) PON, offering two separate wavelength channels per subscriber. A pure WDM PON provides an individual down- and upstream wavelength channel to each user, and thus there is no competition among them and no congestion will occur in the network. However, there is also no opportunity to share capacity among the subscribers, and to use the available network resources in a flexible way.

Introducing a WDM dimension on top of a TDM PON system combines the increased capacity delivered by WDM and the inherent capacity sharing of a TDM PON, and it is an important candidate for next-generation optical access (NGOA) networks. Different hybrid WDM/TDM PON flavors, with a varying remote node architecture, are presented in this paper and compared to each other from several perspectives. Special attention is given to the flexibility aspect by designing an architecture that is able to cope with dynamic bandwidth allocation (DBA) in the time and wavelength domain. Additionally, the considered hybrid WDM/TDM PON architectures are also evaluated from a cost and reach perspective. For the cost evaluation, a 10G technology is considered, taking into account reasonable target costs for the optical components. This evaluation leads to a better understanding of the additional cost for introducing flexibility. The reach calculation is based on the insertion loss of the different hybrid WDM/TDM PON architectures. With the growing interest for long-reach PON, the passive reach of a PON is becoming an important parameter for an architecture selection. Note that a long-reach PON is in favor of several operators that want to reduce the number of central offices in their network, referred to as node consolidation.

The remainder of this paper is organized as follows. In Section 2, different WDM/TDM PON architectures are described, accompanied with a high-level 
evaluation in terms of flexibility, simplicity, insertion loss and security. The motivation for introducing flexibility in NGOA networks is highlighted in Section 3. Further, the needed degree of flexibility in hybrid WDM/TDM PONs is evaluated in Section 4. Finally, Section 5 evaluates the main constraints raised by an increased flexibility, i.e. an increased cost and insertion loss.

\section{Hybrid WDM/TDM PON architectures}

A general architecture of a (flexible) hybrid WDM/TDM PON is shown in Figure 1. Three network parts are indicated between the optical line terminal (OLT) in the central office (CO) and the optical network unit (ONU) at the user side: feeder between OLT and remote node 1 (RN1), distribution between RN1 and RN2, and last mile between RN2 and ONU. In RN2, a passive power splitter (1:N) is installed, which means that RN1 is connected to $M$ TDM PON architectures.

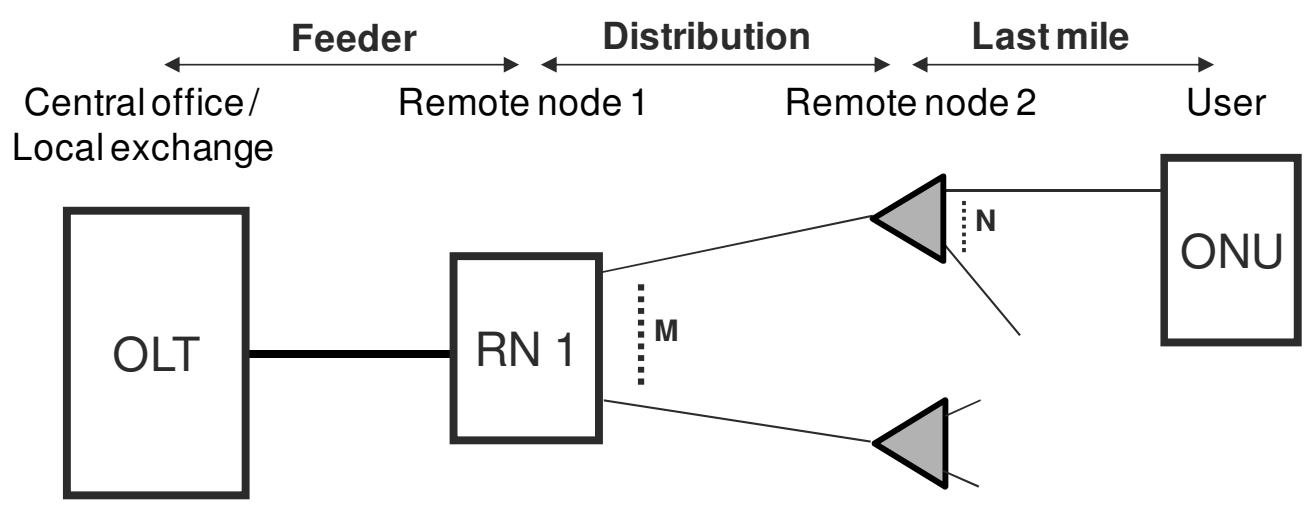

Figure 1: General hybrid WDM/TDM PON architecture

Figure 2 shows the architectures of the considered OLT and ONU. The OLT consists of $W_{u}$ uplink line cards and photo detectors (PD) and $W_{d}$ downlink line cards and distributed-feedback (DFB) lasers. All upstream/downstream wavelengths are (de)multiplexed by using e.g., two arrayed waveguide gratings (AWGs) as wavelength splitter/combiner, and both wavelength bands are put on the same fiber by using a three-port circulator. The ONU contains a three-port circulator to separate upstream and downstream wavelengths. Further, the upstream part of the ONU has an uplink line card and a tunable burst mode transmitter (Tx) for tuning to any desired wavelength. The downstream part has a downlink line card, a classical PD and a tunable optical filter for selecting the desired wavelength. Note that with the current technologies, the realization of 
tunable optical filters with sharp notches, required for hybrid WDM/TDM PONs, can be very expensive, but our believe is that these devices will be available in the future for reasonable cost to be used for access technologies.
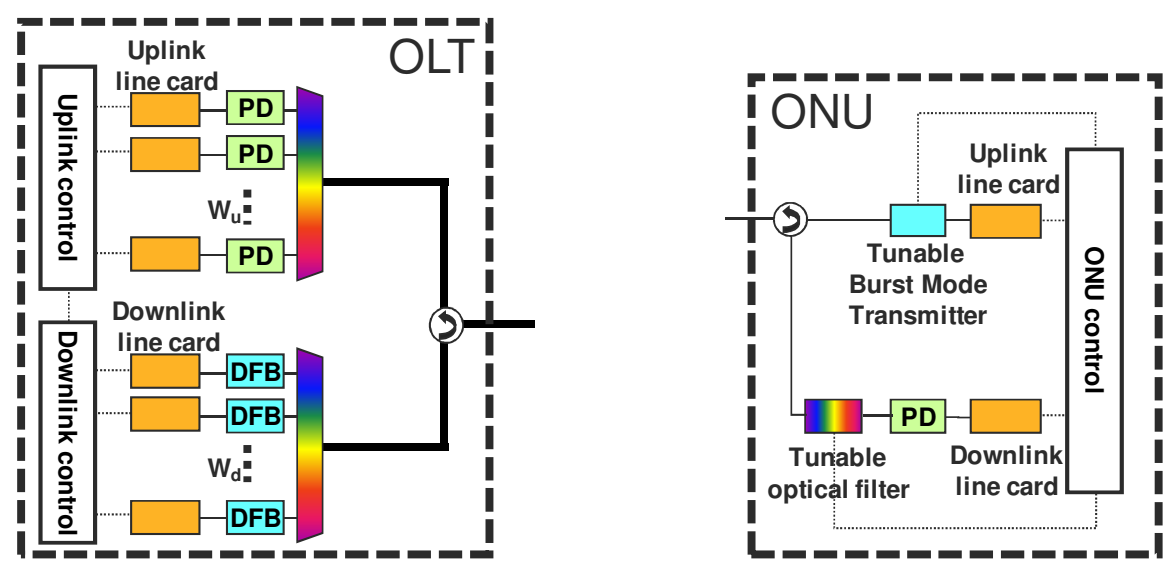

Figure 2: Architecture of OLT (left) and ONU (right)

By varying RN1, three main categories of hybrid WDM/TDM PONs are presented in this section, each with a different degree of flexibility: (1) fully flexible, (2) fully static and (3) partially flexible. Strictly speaking some of the presented hybrid WDM/TDM PON flavors are no longer passive because they may need some (simple) active elements in RN1. However, in these architectures, the data transfer remains optically transparent, and only the control needs opticalelectrical-optical (OEO) conversion. We can also refer to these systems as semipassive, but in general the term PON is still used.

Choosing the best architecture is typically a trade-off between flexibility on one hand, and cost, reach and security constraints on the other hand. In this section, a basic assessment for the different architectures is presented, based on the following criteria: flexibility, simplicity (related to cost), insertion loss (related to reach) and security.

\subsection{Fully flexible architectures}

In the first category, two fully flexible hybrid WDM/TDM PON architectures are presented: a pure broadcast-and select PON and a wavelength-routed PON (see Figure 3). In a fully flexible architecture, each wavelength can simultaneously be routed to any TDM PON (or RN2), and by consequence each TDM PON can be 
reached by any wavelength. These architectures have an inherent broadcast facility, but most variants suffer from higher insertion losses.

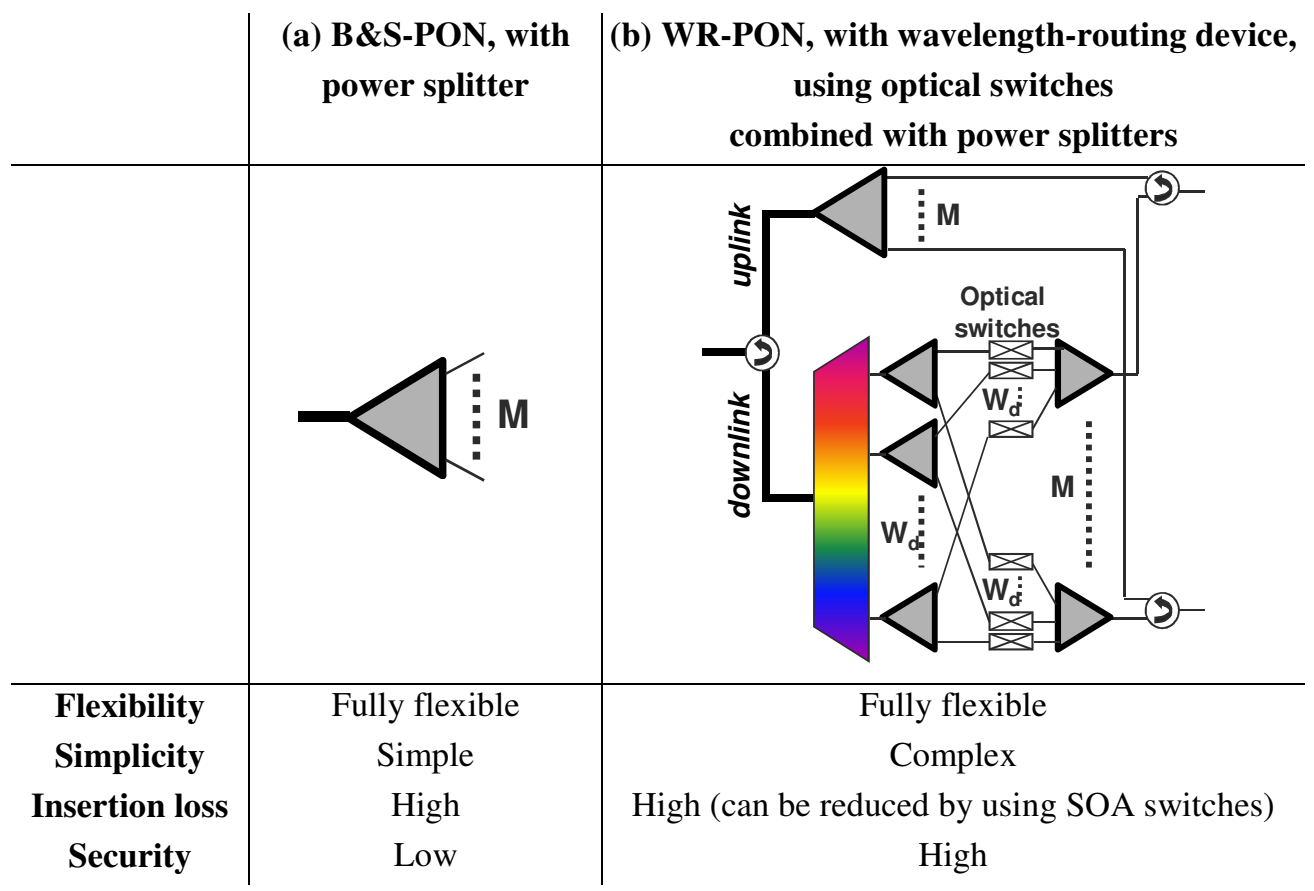

Figure 3: Fully flexible remote node 1 (RN1) variants, resulting in fully flexible hybrid WDM/TDM PON flavors

\subsubsection{Broadcast-and-select hybrid WDM/TDM PON}

In a broadcast-and-select $(\mathrm{B} \& S)$ hybrid WDM/TDM PON, RN1 is a power splitter, and all wavelengths are broadcast to all TDM PONs (or RN2s), and by consequence to all ONUs, as illustrated in Figure 3(a). In this way, each ONU is time shared with the other ONUs with the advantage of having access to a WDM dimension. In the downstream direction, all wavelength channels are broadcast from the local exchange to all the users, without any selectivity in the network itself. The selection is done by the ONU at the user side. In the upstream direction, a wavelength is chosen taking into account the load constraints of the network.

This architecture is very simple (using a legacy power-split optical distribution network) and provides full flexibility. However, it suffers from high insertion losses and it also has a serious security threat as the content of all wavelengths is available to all ONUs. Note that a coherent detection technique at the receiver can deal with the high insertion losses as it allows increasing the optical link budget 
up to $50 \mathrm{~dB}$ (compared to ca. $30 \mathrm{~dB}$ for direct detection techniques in current PON architectures). For an access network, however, coherent detection is still a very complicated and expensive technique, and as such we do not consider it in the remainder of this paper. Currently, coherent detection is investigated for the socalled Ultra Dense WDM PON presented in [5]. This is a pure WDM PON architecture with a 1:1000 split ratio using a fully passive architecture (only using power splitters).

\subsubsection{Wavelength-routed hybrid WDM/TDM PON}

In a wavelength-routed (WR) hybrid WDM/TDM PON, RN1 allows a flexible wavelength routing by adding an active wavelength-routing device (or configurable optical switch) in RN1. An example of a fully flexible wavelengthrouting device is shown in Figure 3 (b), consisting of a WDM splitter, a passive splitter stage, optical switches and a passive combiner stage in the downlink direction. Each TDM PON (or RN2) can get data from all downstream wavelengths, and this is controlled by the optical switches. Each wavelength, on its turn, can be routed to one or more TDM PONs, providing a selected and dynamic multicast environment. In the uplink direction, the data streams from the different TDM PONs (or RN2) are combined through an $M \times 1$ combiner. The uplink and downlink data streams are combined by a three-port circulator. This architectural solution is also referred to as active routing optical access network (ARON) architecture, and is studied in more detail in [6]-[8].

This implementation improves the broadcast nature and security concerns of the B\&S-PON. In general, this architecture also suffers from high insertion losses, but by using fast-switching SOA switches, the high losses due to the couplers and splitters can be partially compensated, but at the cost of a more expensive solution than when e.g. micro-electro-mechanical systems (MEMS) switches are used. In the insertion loss and reach calculations of Section 5, however, we will prove that for a high fan-out case (with e.g. 1000 subscribers per OLT), this architecture, even with SOA switches, is not able to provide a minimum reach of some kilometers. 


\subsection{Fully static architectures}

In the second category, a fully static hybrid WDM/TDM PON architecture with only one variant is presented: a wavelength-split PON (see Figure 4). In a fully static architecture, each wavelength is routed to only one fixed TDM PON (or RN2), and each TDM PON can be reached by only one fixed wavelength. Such an architecture has a low insertion loss and high security.

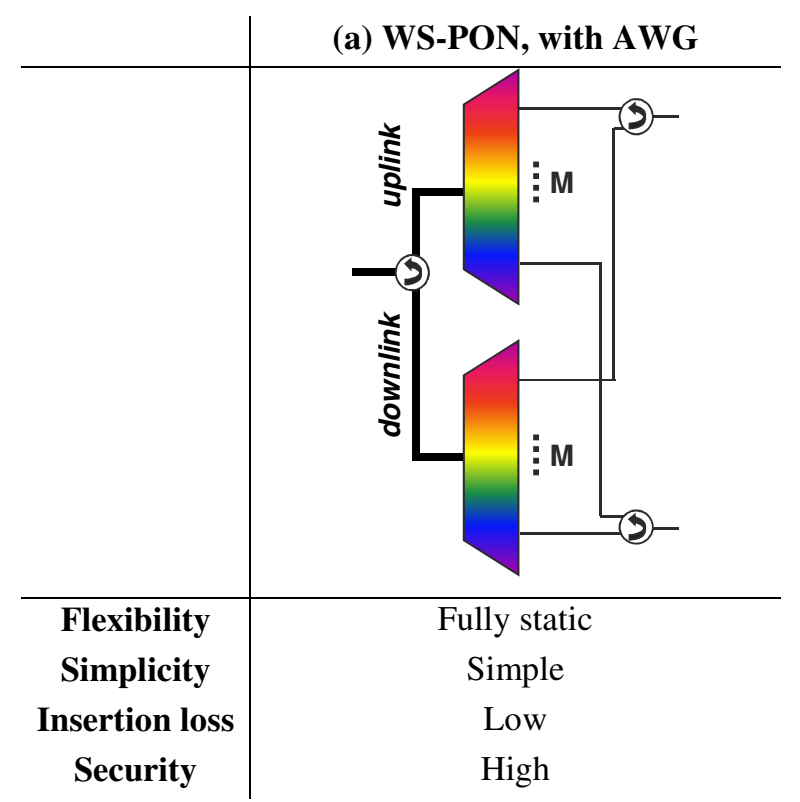

Figure 4: Fully static remote node 1 (RN1), resulting in a fully static hybrid WDM/TDM PON

\subsubsection{Wavelength-split hybrid WDM/TDM PON}

In a wavelength-split (WS) hybrid WDM/TDM PON, a passive wavelength splitter or filter (e.g., AWG) is put in RN1 to distribute different wavelengths to different TDM PONs, as shown in Figure 4, with $W_{u}=W_{d}=M$. This architecture is also extensively discussed in literature, e.g. [9]-[10]. Note that a separate AWG is depicted for the up- and downlink direction, as typically different wavelength bands are used for upstream (e.g. C-band, 1530 - $1565 \mathrm{~nm}$ ) and for downstream (e.g. L-band, 1565 - $1625 \mathrm{~nm}$ ). If the same wavelength band is used, a single AWG can be used for both the up- and downlink part.

As a wavelength splitter has a much lower insertion loss compared to a passive power splitter, this architecture has a longer reach and can support more users. However, the flexibility is very restricted as each wavelength is connected to a fixed TDM PON, this cannot be rearranged with e.g. a changing traffic demand. 


\subsection{Partially flexible architectures}

In the third category, different partially flexible hybrid WDM/TDM PON architectures are presented. These architectures are typically more costly than the fully flexible or fully static counterparts, but they have a higher security and lower insertion loss than the fully flexible architectures, and are, of course, more flexible than a fully static architecture. Often, a trade-off between these different parameters will decide about the best architecture in a specific situation.

In all partially flexible architectures, each TDM PON can be reached by multiple wavelengths. However, each wavelength can reach either multiple or only one TDM PON, and for that reason, we divide these architectures in two main categories: architectures with and without multicasting, respectively.

\subsubsection{Partially flexible architectures, with multicasting}

Three variants of a partially flexible hybrid WDM/TDM PON architecture with multicasting, are discussed in this section (see Figure 5). In a partially flexible architecture with multicasting, each wavelength can be routed to multiple TDM PONs (but not all TDM PONs can be reached by one wavelength at a time). As for all partially flexible architectures, each TDM PON can be reached by multiple wavelengths. The most straightforward way to implement such a partially flexible architecture is attained by combining the B\&S-PON and the WS-PON, i.e. a combination of power splitters and AWGs. However, more complex alternatives can be designed to enhance the flexibility, and two examples using a wavelength selective switch (WSS) instead of an AWG are discussed in this section. Note that all variants are making use of power splitters to offer multicasting. 
(a) B\&S-PON, combined $\mid$ (b) B\&S-PON, combined $\mid$ (c) B\&S-PON, combined

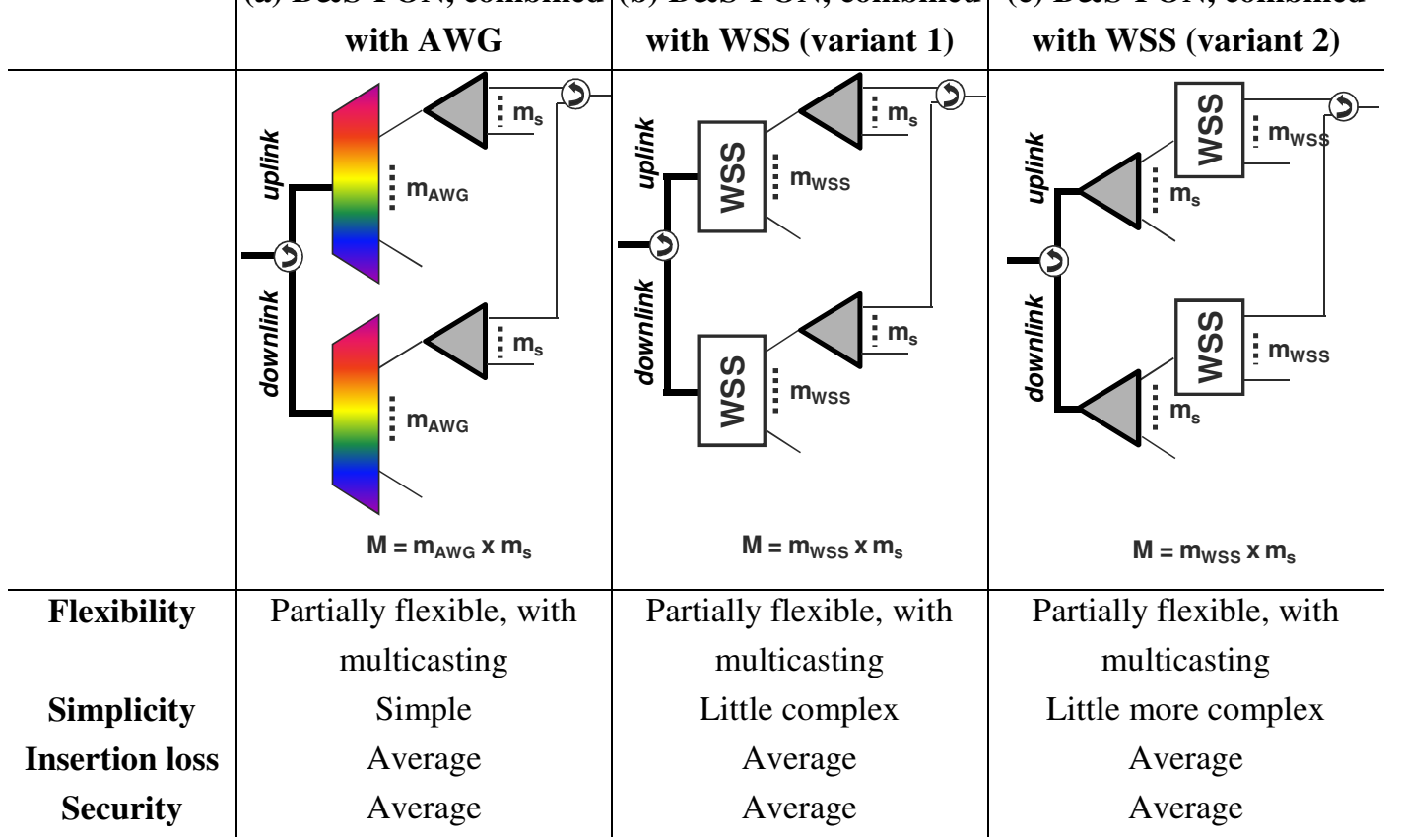

Figure 5: Partially flexible remote node 1 (RN1) variants with multicasting, resulting in partially flexible hybrid WDM/TDM PON flavors with multicasting

\section{Broadcast-and-select hybrid WDM/TDM PON, combined with AWG}

In a B\&S hybrid WDM/TDM PON combined with AWG, RN1 consists of an AWG followed by multiple ( $m_{A W G}$ for the uplink and downlink direction, respectively) power splitters, to distribute one wavelength to different TDM PONs, as shown in Figure 5(a). In this way, a direct combination is made between a flexible B\&S-PON and a non-flexible WS-PON.

By using an AWG, the high power splitter loss as compared to a B\&S-PON is reduced by a factor $m_{A W G}$ (assuming that the total RN1 split factor $M=m_{A W G} \times m_{s}$ is kept constant), and only a low insertion loss of e.g. $3 \mathrm{~dB}$ of an AWG is added. Besides, by including a (smaller) power splitter, some flexibility from a B\&SPON is still available. Both the insertion loss and flexibility are decreasing, when $m_{A W G}$ is increasing, and the trade-off between insertion loss (and reach) and flexibility defines the optimal choice of $m_{A W G}$. Together with the B\&S-PON and WS-PON, this architecture is evaluated in detail in Section 4, to estimate the influence of flexibility on the total amount of used wavelengths.

\section{Broadcast-and-select hybrid WDM/TDM PON, combined with WSS (variant 1)}

In variant 1 of a B\&S hybrid WDM/TDM PON combined with WSS, RN1 consists of a wavelength selective switch (WSS) followed by multiple ( $m_{\text {WSS }}$ for the uplink and downlink direction, respectively) power splitters, as shown in 
Figure 5(b). In this architecture, the (static) AWG from the previous architecture is replaced by a (reconfigurable) WSS [11].

WSSs are generally implemented in MEMS that provide low insertion loss wavelength switching capabilities. A WSS can steer each wavelength channel present on its common input port towards one of its output ports. In contrast to an AWG, this output port is no longer static, but can be selected by the WSS, enhancing the flexibility of RN1. Off-the-shelf WSS can have the functionality of $1 \times 2,1 \times 4$ or $1 \times 8$ switching. A WSS has the capability of steering one wavelength from an output port to another one if the users attached to the concerned output port do not require the service of that wavelength anymore. From a reach and security perspective, there is no much difference between using an AWG and WSS (e.g., we can assume that the insertion loss of a WSS is in the same order of magnitude as the loss of an AWG). The main disadvantage of using a WSS, however, is its comparatively high cost, as discussed in Section 5.

\section{Broadcast-and-select hybrid WDM/TDM PON, combined with WSS (variant 2)}

In variant 2 of a B\&S hybrid WDM/TDM PON, combined with WSS, RN1 consists of a power splitter followed by multiple $\left(m_{s}\right.$ for the uplink and downlink direction, respectively) WSSs, as shown in Figure 5(c). In this architecture, the power splitter and WSS are changed from order compared to the previous architecture. This hybrid WDM/TDM PON flavor is discussed in detail in [12]. As the power splitter is put in front of the WSS, the flexibility is further enhanced, as RN1 can now do a limited (flexible) multicasting. The same wavelength can be multicast to $m_{s}$ different output ports of $\mathrm{RN} 1$, provided they are attached to different WSS modules. The reach and security aspects are comparable to the previous partially flexible architectures. The main disadvantage of this second variant of a WSS-based B\&S-PON architecture, compared to the first variant, is its higher cost due to the use of a higher amount of WSSs $\left(m_{s}\right.$ instead of 1 for the uplink and downlink direction, respectively).

\subsubsection{Partially flexible architectures, without multicasting}

Two variants of a partially flexible hybrid WDM/TDM PON architecture without multicasting, are discussed in this section (see Figure 6). In a partially flexible architecture without multicasting, each wavelength can be routed to one TDM PON only at a time (i.e., no multicasting of a wavelength among multiple TDM 
PONs is allowed). However, each TDM PON can still be reached by multiple wavelengths. In general, these architectures are extended WS-PONs, using an AWG as basic element. On the other hand, they do not make use of power splitters because no multicast functionality is needed, which means that their insertion loss is much lower than for the partially flexible solutions with multicasting.

As multiple wavelengths can be offered to one TDM PON, there is the flexibility to adapt the number of wavelengths per TDM PON according to the traffic demand. However, since each wavelength can only be routed to one TDM PON simultaneously, this architecture is mainly of importance if the number of wavelengths ( $W_{d}$ and/or $W_{u}$ ) is larger than the number of TDM PONs $M$, because otherwise, e.g. if the number of wavelengths is equal to $M$, one or more TDM PONs cannot be reached from the moment another TDM PON is served by two or more wavelength.

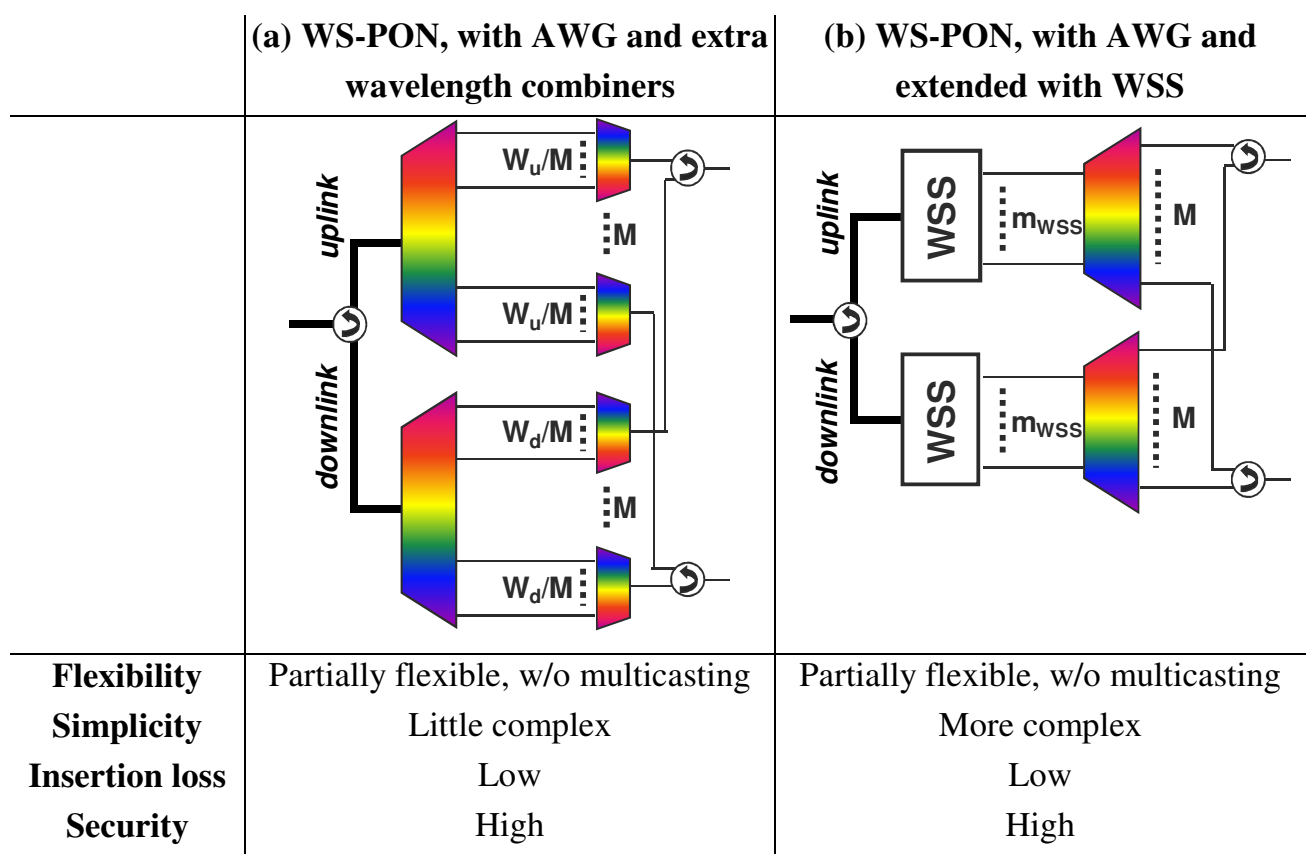

Figure 6: Partially flexible remote node 1 (RN1) variants without multicasting, resulting in partially flexible hybrid WDM/TDM PON flavors without multicasting

\section{Wavelength-split hybrid WDM/TDM PON, with extra wavelength combiners}

In a WS-PON with extra wavelength combiners, RN1 consists of an AWG followed by $M$ wavelength combiners to offer multiple wavelengths per TDM PON, as shown in Figure 6(a). The (average) number of down- and upstream 
wavelengths provided to each TDM PON is equal to $W_{d} / M$ and $W_{u} / M$, respectively. From these formulas, it is also clear that this architecture only makes sense if $W_{d}$ (or $\left.W_{u}\right)>M$. If $W_{d}$ (or $\left.W_{u}\right)=M$, this architecture is reduced to the WSPON from Figure 4.

\section{Wavelength-split hybrid WDM/TDM PON, extended with WSS}

In a WS-PON extended with WSS, RN1 consist of a WSS followed by an AWG, as shown in Figure 6(b). Compared to a WS-PON, a WSS enhances the wavelength reconfigurability among the subscribers according to their traffic demand. In particular, the WSS can select which wavelength to be routed to which input port of the AWG. Combing a WSS with an AWG provides partial flexibility of choosing multiple (with a maximum equal to the number of WSS output ports, i.e. $\left.m_{W S S}\right)$ wavelengths to be routed to a particular output port of the AWG. This architecture is also presented in [12].

As only a maximum of $m_{W S S}$ wavelengths can reach a particular TDM PON (or $\mathrm{RN} 2$ ), we can increase the flexibility of the proposed architecture by increasing the number of output ports per WSS. This brings a cost versus flexibility tradeoff issue in the architecture design. As for all WSS-based PONs, there is a costly WSS required, but just as in variant 1 of the B\&S hybrid WDM/TDM PON architecture combined with WSS, this solution only requires one WSS for upstream and downstream, respectively.

\section{Motivation to add flexibility in access networks}

The flexibility to offer any bandwidth (in the limit of the physical bit rate) to anybody could be a major advantage for NGOA networks. A hybrid WDM/TDM PON with DBA in the time and wavelength domain can combine the virtues of both the TDM and WDM solutions. The introduction of a WDM layer allows for an increase of both the number of users and total data rates, while the granularity of the TDM layer on top of the WDM layer offers a better bandwidth usage, scalability and upgradeability than a pure WDM PON. By combining WDM and TDM, a lot of demands can coexist on the same network infrastructure in a very efficient manner. This is a strong advantage when compared to other systems.

From the OLT, multiple TDM PONs can be set up, each at a specific wavelength. Each TDM PON serves a set of users, and within this set, the capacity is shared. By means of wavelength selection or routing, the number of users within the set 
can be varied, and thus the capacity offered per user can be varied. Hence a flexible hybrid WDM/TDM PON can offer capacity-on-demand, and the congestion probability can be significantly reduced compared to a static wavelength configuration. The main advantages of a flexible hybrid WDM/TDM PON are highlighted in the next subsection. Then, the most important options for implementing this flexibility are described, and finally the constraints that come into the picture are treated.

\subsection{Advantages offered by flexibility}

If flexibility can be added at RN1, so that any wavelength can be steered to any or a part of the passive power splitters in RN2, it is possible to improve the network performance like bandwidth utilization and delay, the energy efficiency of the overall network operation, the extensibility of the network, as well as the smooth migration to next-generation network technologies. In the next subsections, we give a brief account of the four most striking features of this architecture that separate it from most of the other PON architectures, i.e., network performance, energy efficiency, network extensibility and the network migration.

\subsubsection{Network performance}

A (partially) flexible network architecture naturally provides the means to have an improved network performance (like bandwidth utilization and delay) by applying advanced resource allocation techniques. Combing the WDM and TDM layer allows a better installation of a DBA scheme due to the wavelength allocation flexibility, and it enjoys a better statistical-multiplexing gain and bandwidth-usage efficiency. The use of power splitters in the proposed hybrid WDM/TDM PONs allows us to broadcast wavelengths to multiple user groups, and by using DBA protocols, we can select users per wavelength on the basis of their network load. As we use a passive power splitter, a wavelength splitter (or filter), or an active optical switch (e.g., SOA, MEMS, WSS), it keeps the data layer transparent in all the remote nodes, and hence reduces the overall network processing time and data transfer delays, and eventually supports maintaining more stringent quality of service requirements. Efficient bandwidth utilization also leads to energy efficiency as we have to use a smaller number of wavelengths, and by consequence less line cards, for attaining the same network performance. 


\subsubsection{Network extensibility}

The network extensibility means the efficient deployment strategies that the hybrid WDM/TDM PON architecture can promote while constantly expanding the network by the incremental installation of network equipment according to the connectivity demand of the end users. Due to the inherent flexibility, the proposed architecture can be used for green- or brown-field installation where the network connectivity demand can change and evolve over time. The example provided in Figure 7 explains the concept in a more illustrative way.
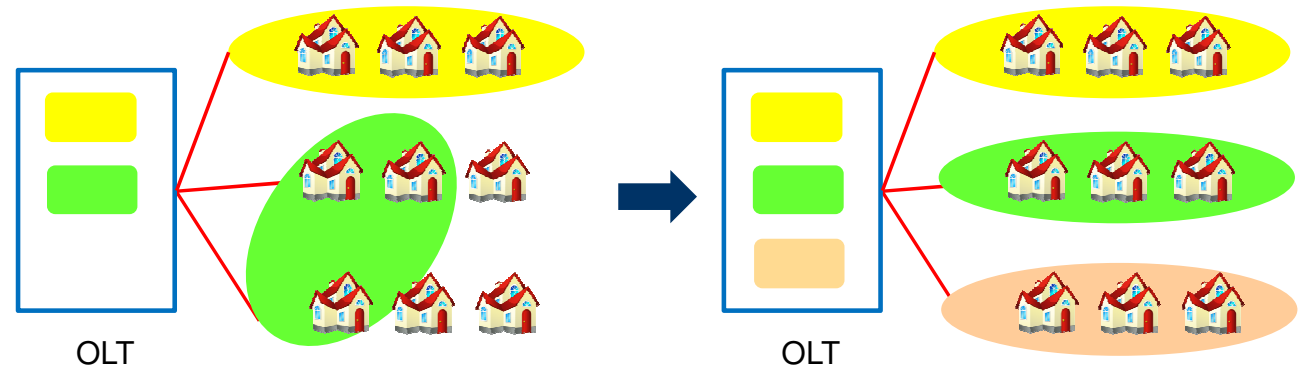

Figure 7: Illustration of a network extensibility scenario for flexible hybrid WDM/TDM PONs

Figure 7 provides an example where the first remote node has physically three possible splitting stages. Now, initially as shown in the left part of Figure 7, the number of users connected to the second and third splitting-point is less than optimal. Therefore due to the wavelength flexibility of our architecture, we can allocate a single wavelength (green for example) to all the users attached to the second and third splitting-point as depicted in Figure 7 and therefore use just two transceivers and line cards at the OLT. As the demand increases, and more subscribers are added to the relevant splitting-points, the OLT can have added line cards to fulfill the demands of the expanded network, and the wavelengths at the user premises can be reallocated to cope with the individual traffic needs as shown in the right-hand part of the Figure 7. This provides a means for the operator for a smooth and incremental expansion of the network according to the demand, while keeping the basic network infrastructure deployed in the field undisturbed. 


\subsubsection{Energy efficiency}

Our proposal for energy efficiency has a similar argument as provided for the network extensibility. An illustrative example is provided in Figure 8 to demonstrate this. The left part of Figure 8 shows that over time, users who require almost no services (e.g., business users during the night time hours) can be reconnected to a particular wavelength. In this scenario, the wavelength can be reallocated as shown in the right-hand part of Figure 8 and this provides a mean to turn off some of the OLT transceivers as well as line cards, to enhance energy savings at the OLT over time. This might help the operator to build its network greener. The same scheme also depicts the possibility of dynamic allocation of wavelengths amongst users according to their traffic needs.

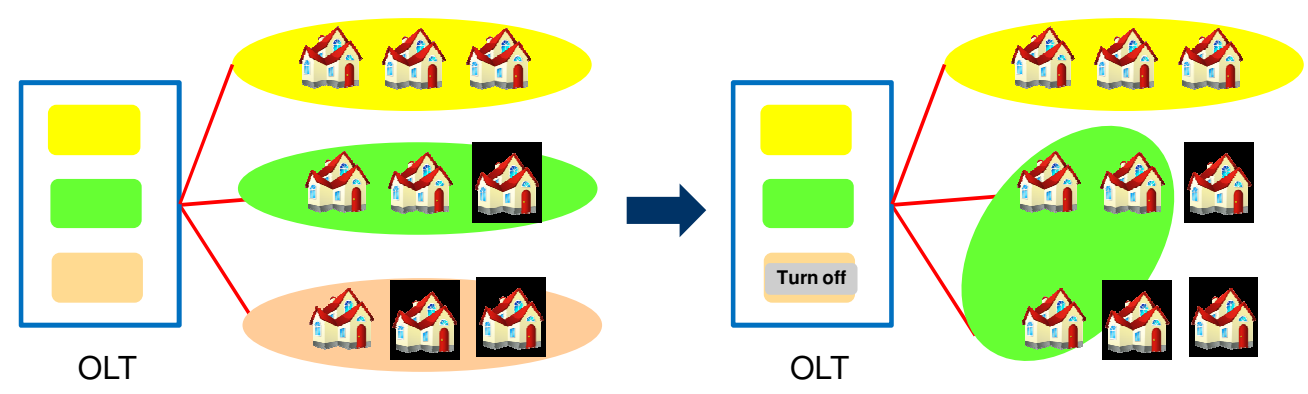

Figure 8: Illustration of an energy efficiency scenario for flexible hybrid WDM/TDM PONs

\subsubsection{Network migration}

Our proposal for network migration corresponds to an efficient migration strategy that the proposed architecture can support during e.g. a technology upgrade. In this way, co-existence of a new technology with the legacy system is made possible. Figure 9 provides an example where some subscribers move from a legacy $1 \mathrm{G}$ wavelength service to a new $10 \mathrm{G}$ wavelength service. In this case, by reallocation of wavelengths as shown in the right part of the Figure 9, the network demands can be accomplished by just having one OLT transceiver that supports a 10G wavelength. This strategy allows a more smooth transition of the network during the migration phase. 


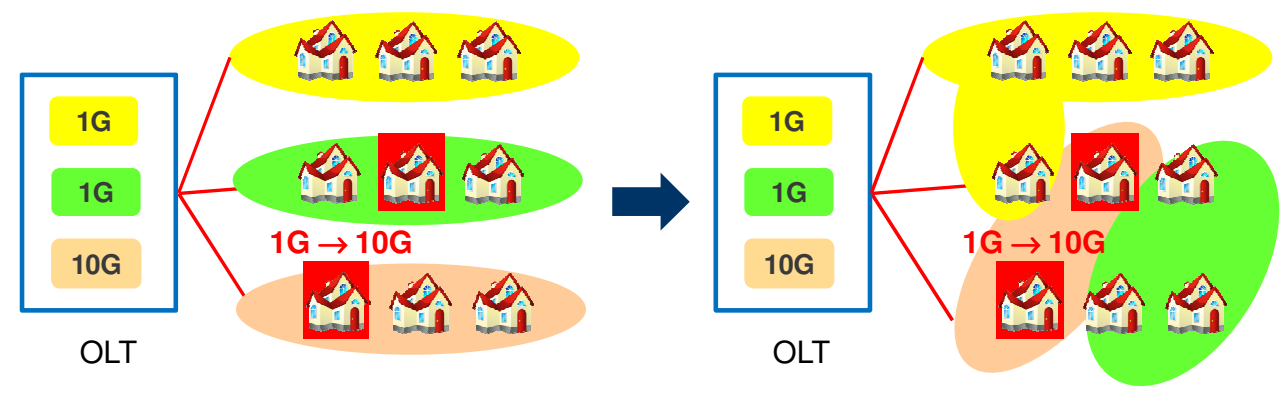

Figure 9: Illustration of a network migration scenario for flexible hybrid WDM/TDM PONs

\subsection{Options to implement flexibility}

To capture the above advantages, one needs to carefully assess the flexibility options. In general, there exist two different kinds of flexibility depending on the architecture choice.

\subsubsection{Short-term flexibility}

Short-term flexibility means in the limit that every medium access control (MAC) frame can individually be allocated to a certain ONU. However, typically a group of frames are allocated per ONU as otherwise the DBA scheme will be very inefficient due to the huge overhead for accessing each frame individually. Such a group of frames is called a burst, and in this way we can also refer to short-term flexibility as burst-by-burst flexibility. Such a burst-by-burst flexibility is possible with a fully-passive, B\&S-PON-based architecture, where every wavelength is multicast to all (pure B\&S-PON, Figure 3(a)) or multiple (B\&S-PON with AWG, Figure 5(a)) TDM PONs. The OLT scheduler as well as the MAC protocol can then determine on the fly what should be the best possible wavelength mapping, and the burst selection is made at the ONU. However, as will be shown in Section 5 , these architectures are experiencing the highest insertion losses. All other listed hybrid WDM/TDM PON architectures have a switching device, consisting of active optical components, in the first remote node. Such a switching device is controlled in the OLT, and a separate control channel between the OLT and remote node is needed to control the switching device in the remote node. Further, active optical networks (AON), e.g. based on Ethernet switches, can also deliver this maximum flexibility. These networks, however, need a lot of active electronic equipment in the field which limits their reliability and power efficiency. Moreover, in every node, optical-electrical-optical $(\mathrm{O} / \mathrm{E} / \mathrm{O})$ conversion is needed 
which negatively effects delay and jitter. Nevertheless, AONs have their advantages in some specific cases and are currently used in several municipality networks in Europe, but they are out of scope for this paper.

As the wavelength allocation can be reassessed per burst basis, burst-by-burst flexibility eventually leads to the best possible resource utilization in the most flexible manner possible. However, not all technical solutions considered in this paper are suited for delivering this high flexibility granularity, and moreover some of the described advantages offered by flexibility, like network extensibility and migration, do not need such a short-term flexibility for achieving large gains.

\subsubsection{Long-term flexibility}

Long-term flexibility means that the network resources are reallocated on a longer time frame, like e.g. a daily, monthly, etc. basis. This is possible with all described (partially) flexible architectures, and the switching configuration of the remote node is only changed when new users (ONUs) join the network, or when the operator upgrades its technology (bit rate etc.) for the existing users, or when the users modify their contract to enhance their service level agreement (SLA). We need to keep in mind that for the (partially) flexible remote nodes requiring electronic control, a dedicated control channel (separate wavelength or out of band signaling) from the OLT to the first remote node is needed to remotely implement these changes. The OLT can as well employ traffic pattern learning techniques to learn the daily traffic pattern from each ONU to implement the power saving scheme as described above. As some important flexibility advantages in an access network, like network extensibility and network migration, are long-term issues, it is advised to design a network architecture that can deal with long-term flexibility. The ability to reconfigure the network remotely (instead of manually) without any network interruption, is the main requirement for such a network architecture. In this way, important operational savings are possible.

\subsection{Constraints raised by enhanced flexibility}

Adding extra flexibility in the access network comes also at the cost of other drawbacks, which have to be carefully tackled. The most important constraints are 
the cost of the architecture, the insertion loss (and related reach) and security issues.

\subsubsection{Cost}

Contrary to core networks, the access network is shared by only a few subscribers (e.g., up to 1000), and this limited cost sharing means that sophisticated and expensive network technologies (like e.g. OPS) are excluded for an access network. In the comparison of different hybrid WDM/TDM PON architectures, the cost aspect always has to be kept in mind, and will play a crucial role in the final acceptance of a certain solution. Both capital expenditures (CapEx) and operational expenditures (OpEx) are very relevant in the final technology choice.

\subsubsection{Insertion loss and reach}

As already mentioned in the previous subsection, a fully-passive broadcast-andselect architecture can deliver a high flexibility, but when only passive splitters are used, high insertion losses are experienced, leading to a limited reach. Today's GPON and EPON systems are also based on passive power splitters, but their application area is typically limited to 1:32 or 1:64 power splits, and a reach of the order of $20 \mathrm{~km}$. Many operators attach great importance to node consolidation, meaning that they want to reduce the number of central offices. In this way, longreach optical access networks, with a high fan-out (e.g. up to 1000) are required. This means that extra attention has to be given to the total insertion loss of the proposed architectures.

\subsubsection{Security}

For some access network architectures, security issues come into the picture due to the general broadcast nature in the downlink direction. If the final data selection is made at the ONU (like in a broadcast-and select architecture), evil users can sniff the traffic of their neighboring users and due to the shared access a rough user can even disrupt the entire transmission. To avoid this, advanced security algorithms are proposed and adopted in current GPON and EPON implementations. For NGOA, the security should be no worse than in currently deployed GPON/EPON systems, i.e., common security risk shared among $32-64$ users due to the low fan-out in the broadcast domain. However, if a fan-out of 
1:1000 is required, security risk can further be increased for a fully-passive broadcast-and-select architecture.

\section{Evaluation of flexibility in hybrid WDM/TDM PONs}

As described in Section 2, the proposed hybrid WDM/TDM PONs have a different degree of flexibility, going from fully static, over partially flexible to fully flexible architectures. The more flexible architectures, however, are either more expensive, experience a higher insertion loss or are less secure. A question that arises is if a fully flexible architecture is really needed, or if a partially flexible architecture already can serve the advantages listed in Section 3. An important assessment parameter is the number of needed wavelengths at a certain offered ONU load. For a fully flexible architecture, it is clear that the number of needed wavelengths can be optimally minimized, but how big is the gain of a fully flexible architecture compared to a partially flexible one, and what is the minimum degree of flexibility required to have a significant advantage of the offered flexibility. This section gives an answer to the above questions.

This section starts with a short discussion between the evaluation of short-term and long-term flexibility. Then an overview of possible MAC protocols for hybrid WDM/TDM PONs is given. Although the flexibility is introduced by the architectural design, it is of importance to develop a well-suited MAC protocol that optimally exploits the offered flexibility in terms of dynamic wavelength allocation. Finally, based on a basic simulation environment, the influence of flexibility on the wavelength usage and general performance parameters like bandwidth utilization and delay is investigated by varying the flexibility of the network architecture.

\subsection{Short-term versus long-term flexibility evaluation}

A flexible architecture offers short-term flexibility gains like an improved network performance and energy efficiency, and long-term flexibility gains like network extensibility and network migration. In general, more flexible architectures will offer both better short-term and long-term flexibility.

The quantitative assessment of short-term flexibility gains (like network performance) can easily be done in terms of wavelength usage, bandwidth utilization and delay. A qualitative assessment of long-term flexibility gains can 
be made but the real quantitative assessment of the long-term flexibility depends upon the assumption of realistic network traffic patterns for now and in the future, and upon a realistic scenario which predicts how and when customers will be willing to migrate etc. It is needless to stress that these traffic patterns and scenarios may not be the most realistic and easy to predict.

It is hard to realistically quantify the gains of long-term flexibility. However, they will offer a great opportunity for an operator to design an extensible and a migration friendly architecture. The long-term flexibility assessment is out of the scope of this paper, but the short-term flexibility assessment already gives a good indication of the general flexibility capabilities of the different architectures.

\subsection{MAC protocols for hybrid WDM/TDM PONs}

For the described architectures in Section 2, a suitable MAC protocol is needed to manage the time and wavelength allocation and scheduling. The problem of bandwidth scheduling can be approached in fundamentally two different ways [13]: a) Separated time and wavelength assignment, and b) Joint time and wavelength assignment. Joint time and wavelength assignment is a multidimensional scheduling approach which is more complex, but it is an efficient and scalable approach of upstream scheduling and wavelength assignment (USWA). There exist two USWA approaches [13]: offline and online. In the offline approach [14], the OLT waits until it has received all the reports from the ONUs (or part of them [15]) and then it performs some algorithm to find the best USWA scheme for the corresponding grants. In the online approach [16], upon the arrival and processing of a report from an ONU, the OLT immediately decides on the USWA for the corresponding grant.

For this paper, we have used an offline joint time and wavelength assignment scheme based MAC protocol, comparable to the protocol proposed in [8], to investigate the optimal degree of flexibility in hybrid WDM/TDM architectures. The offline approach has very less implementation complexity and can address fairness and QoS issues among different ONUs. In addition to this, in offline algorithms the OLT would wait until the report messages from all ONUs have arrived and then try to arrange upstream scheduling in an optimal way, thus minimizing void formation, wavelength switching and wavelength use. Note that online algorithms can be useful for further optimizing the MAC protocol when 
short-term flexibility for very delay-sensitive applications is needed. For the purpose of this paper, however, the simpler offline approach completely fulfils our needs.

\subsection{Simulation setup and evaluated hybrid WDM/TDM PON variants}

We have simulated a hybrid WDM/TDM PON with $M$ TDM PONs, consisting of $N$ ONUs (corresponding to a hybrid WDM/TDM PON with $M \times N$ ONUs). From the access side, packets arrive at the ONU from a user connected to that ONU. Packets are buffered in the ONU until the ONU is allowed to transmit them to the OLT. In our model, we consider $R_{D}$ to be the data rate of the access link from a user to an $\mathrm{ONU}$, and $R_{U}$ to be the date rate of an upstream channel from an ONU to the OLT. The maximum distance between OLT and ONU is $100 \mathrm{~km}$. We have chosen $M=16, N=4$, and $R_{U}=1 \mathrm{Gbps}$ and $R_{D}=R_{U} / N=250 \mathrm{Mbps}$. We have generated packets in the form of Ethernet frames (64 to1518 bytes) and packets arrive at each ONU from the end user. The buffer size at each ONU is limited to $10 \mathrm{MB}$. The simulated user traffic is self-similar by aggregating $S=32$ substreams [17], each consisting of alternating Pareto-distributed on/off periods, with a shape parameter of 1.4 for the on period and a shape parameter of 1.2 for the off period. In the on period, the packet arrivals are exponentially distributed with a mean arrival rate $A r$ (in b/s). The variable traffic load can be produced by varying $A r$ and the location parameter for the on and off period. We vary $\operatorname{Ar}$ as $0.009 \Phi$, where $\Phi(\mathrm{Mb} / \mathrm{s})$ is the TDM PON Load. Note that the tuning and switching times of the components were neglected as they are expected to lead to the same performance degradation for all configurations. Also, the performance degradation can be handled with a well suited MAC protocol [18], but this is out of the scope of this paper.

We have assumed that there are 16 wavelengths available for each OLT, and RN1 and RN2 provide a 1:M (1:16) split and a 1:N (1:4) split, respectively. Five different variations are given for a partially flexible B\&S-PON combined with AWG, each with a varying degree of flexibility in RN1 by varying the values for $m_{s}$ and $m_{A W G}$, while keeping $M\left(=m_{s} \times m_{A W G}=16\right)$ constant (as shown in Figure 10). The five architectures are indicated by the term group $\mathrm{x}$, where $\mathrm{x}=m_{s}$ indicating the number of TDM PONs that can share the same wavelength, or the number of wavelengths that can be used by one TDM PON (or RN2). For two 
extreme cases, this architecture is reduced to the broadcast-and-select PON $\left(m_{A W G}=1\right.$, Figure 10(a)) and wavelength-split PON $\left(m_{s}=1\right.$, Figure 10(e)). The broadcast-and-select PON of Figure 10(a) was discussed in Section 2.1.1 and also depicted in Figure 3(a). It provides full flexibility and any of the subscribers can use any of the available wavelengths, i.e. 16 in the Figure 10. The architecture of Figure 10(b-d) was described in Section 2.3.1 and similar to the one in Figure 5(a). In Figure 10(b-d), only 8, 4 or 2 specific wavelengths can be used by a TDM PON or the group of ONUs sharing the last mile passive splitter in RN2. Therefore, any wavelength can only be shared by a limited number $(8,4$ or 2$)$ of TDM PONs. The wavelength-split PON of Figure 10(e) was presented in Section 2.2.1 and on Figure 4. It has no flexibility, as all ONUs can only use their dedicated wavelengths.

Note that in both Figure 4 and Figure 5(a), a separate AWG was depicted for uplink and downlink. For simplicity reasons, however, Figure 10 shows an architecture with uplink and downlink wavelengths in the same band so that only one AWG can be used. In the architectures of Figure 10, an increasing flexibility corresponds to an increasing insertion loss (and by consequence a shorter reach).

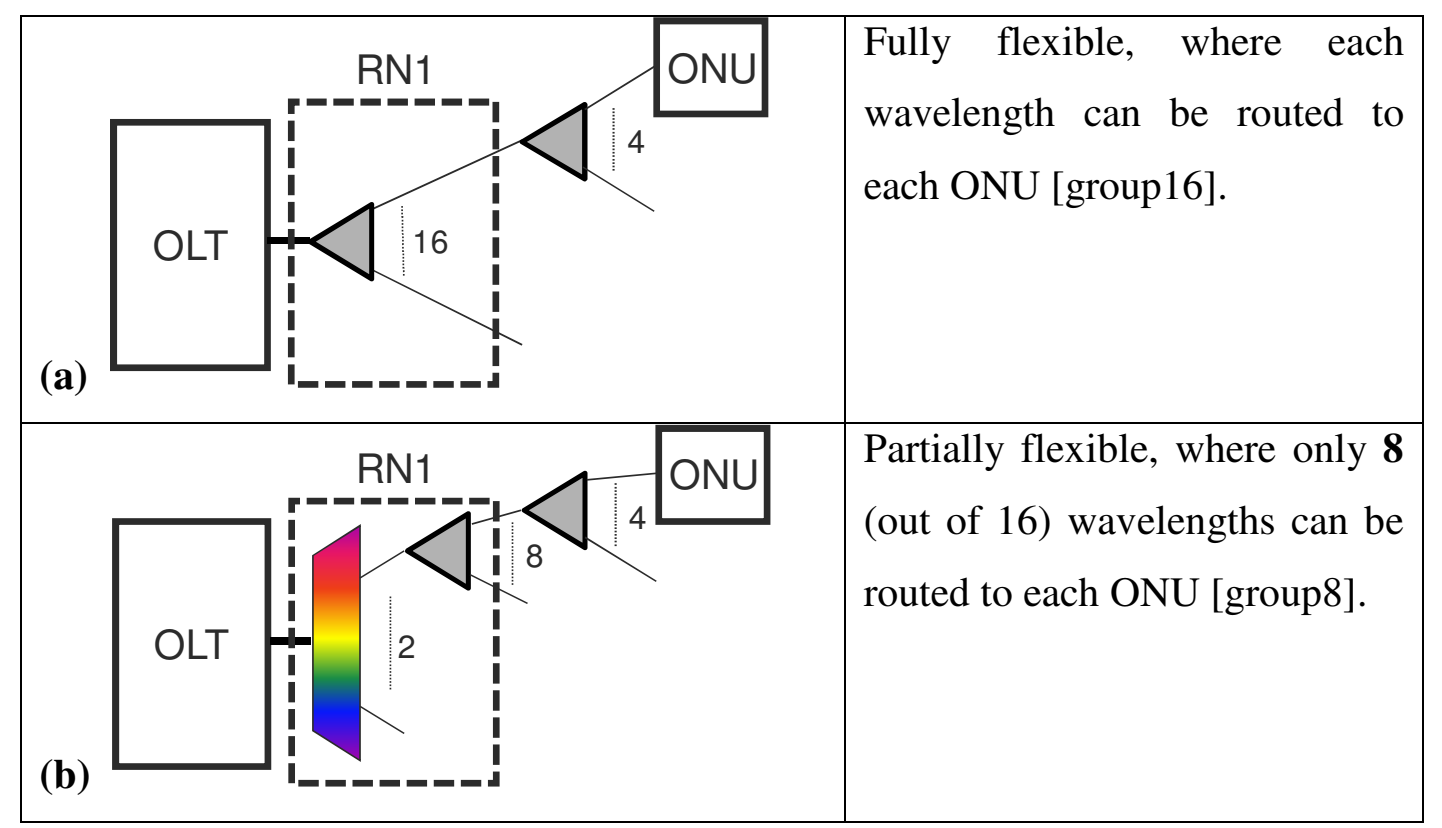




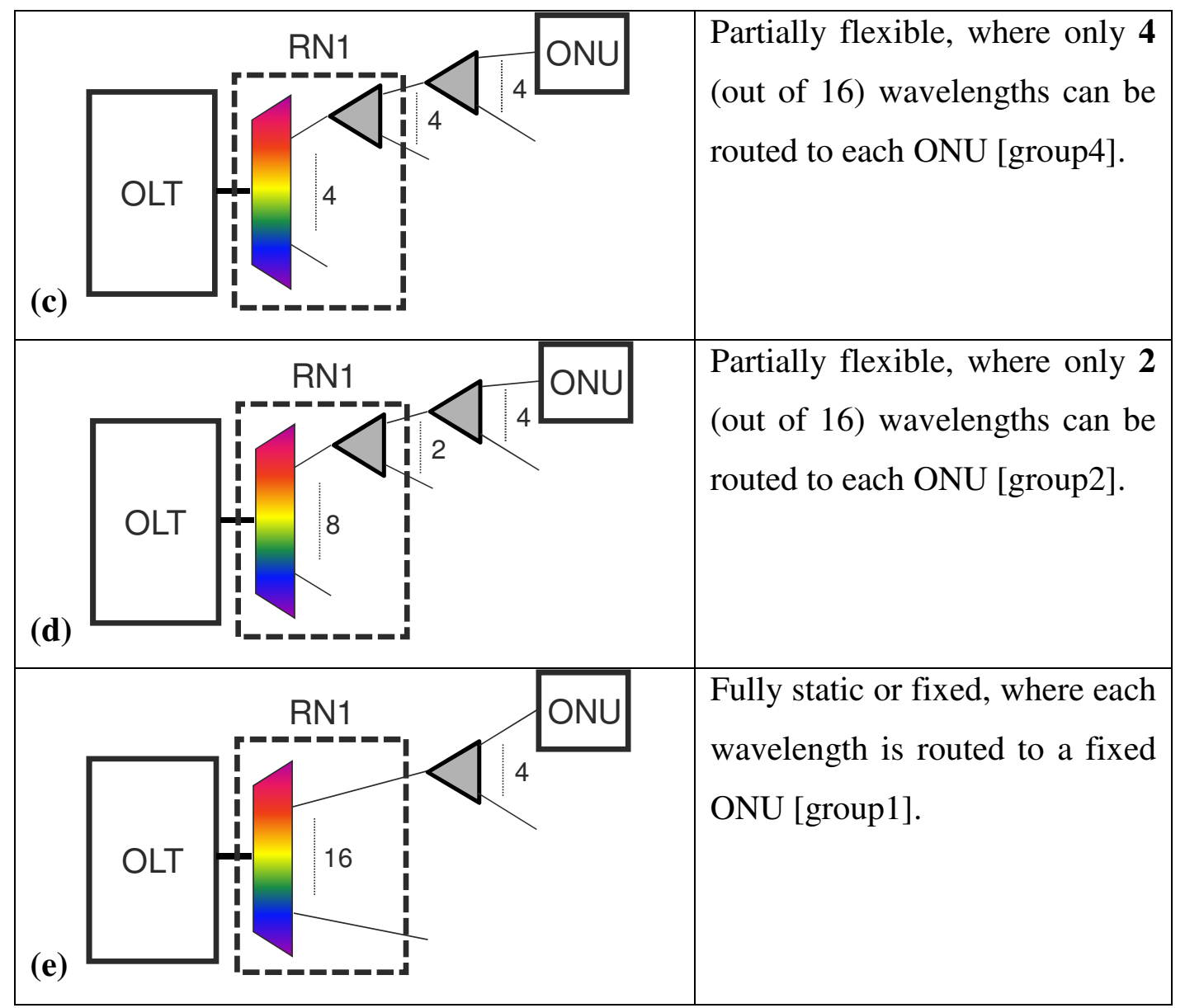

Figure 10: Hybrid WDM/TDM PON flavors based on AWGs and power splitters with a different degree of flexibility in RN1, going from (a) fully flexible, (b-d) partially flexible, to (e) fully static

\subsection{Influence of flexibility on the network performance}

This section shows and discusses the flexibility results for the network performance, in terms of wavelength usage, bandwidth utilization and delay.

Figure 11 and Figure 12 show the average and maximum number of wavelengths, respectively, required to satisfy the overall offered TDM PON (or RN2) load in the five scenarios from Figure 10. In Figure 11, we notice a faster increase of the average number of wavelengths for group2 from a load of ca. 0.4. This can be explained as for group2, in the beginning, every TDM PON can typically be served by one wavelength, resulting in a total of eight needed wavelengths. With higher loads, multiple (and not only one by one) TDM PONs will need a second wavelength, explaining the faster increase from that point. 


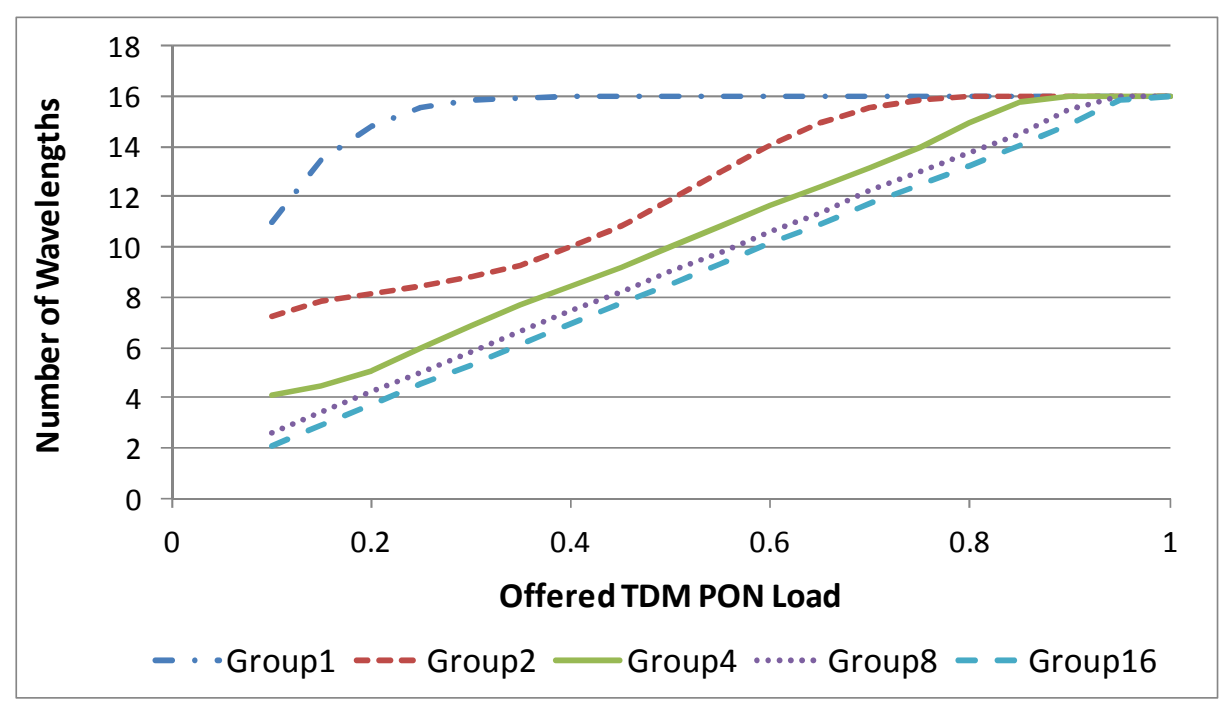

Figure 11: Average number of wavelengths required in function of the offered TDM PON or RN2 load, for five hybrid WDM/TDM PON variants with a different degree of flexibility in RN1

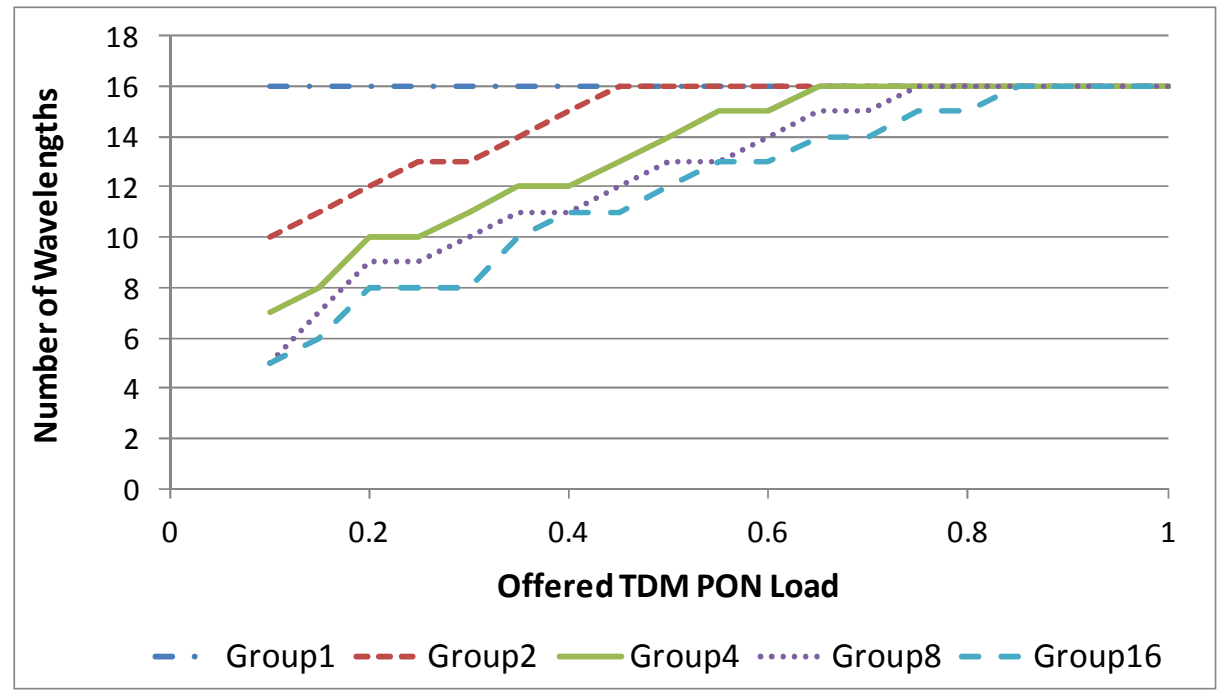

Figure 12: Maximum number of wavelengths required in function of the offered TDM PON or RN2 load, for five hybrid WDM/TDM PON variants with a different degree of flexibility in RN1

Figure 13 represents the bandwidth utilization, in terms of channel underutilization, for the five scenarios from Figure 10. It is obvious that sharing a higher number of TDM PONs by the same wavelength (e.g. group16) leads to a more efficient utilization of the wavelength channels or a lower channel underutilization. At low loads the channel under-utilization is decreasing from $85 \%$ for group1 to $20 \%$ for group16, and at high loads it is decreasing from $20 \%$ to $3 \%$. 


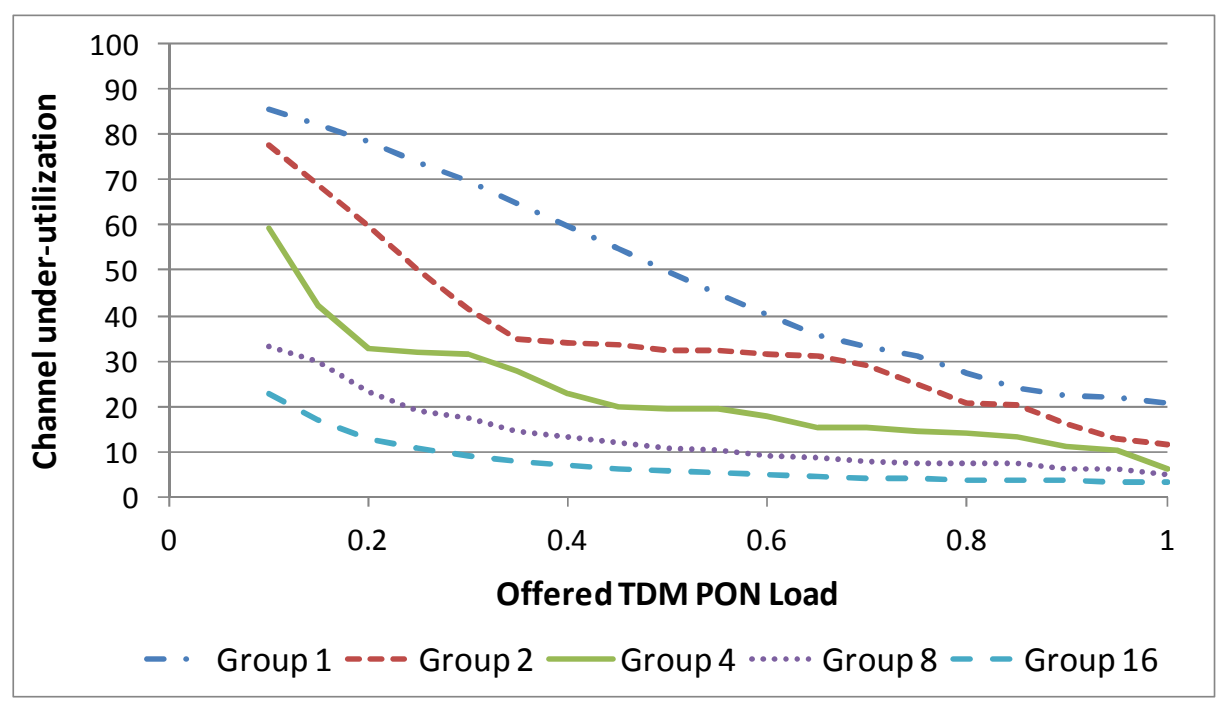

Figure 13: Channel under-utilization in function of the offered TDM PON or RN2 load, for five hybrid WDM/TDM PON variants with a different degree of flexibility in RN1

Figure 14 shows the average delay for the five scenarios from Figure 10. The lower bound of the delay is equal $1.5 \mathrm{~ms}$ for the considered reach of $100 \mathrm{~km}$ (i.e. $3 / 2$ of the cycle time [19]-[20] or $3 / 2$ of the maximum round-trip time of the PON). The simulated traffic has a low peak-to-average load ratio of approximately 1.15 [i.e. $(A r \times N \times S) / \Phi=0.009 \times 4 \times 32$ ], and thus even for a completely static configuration (group1), the delay values are not very high at high load. From the simulation results, we can see that a partially flexible architecture (like group2) provides much gain in delay performance with only a small addition of flexibility.

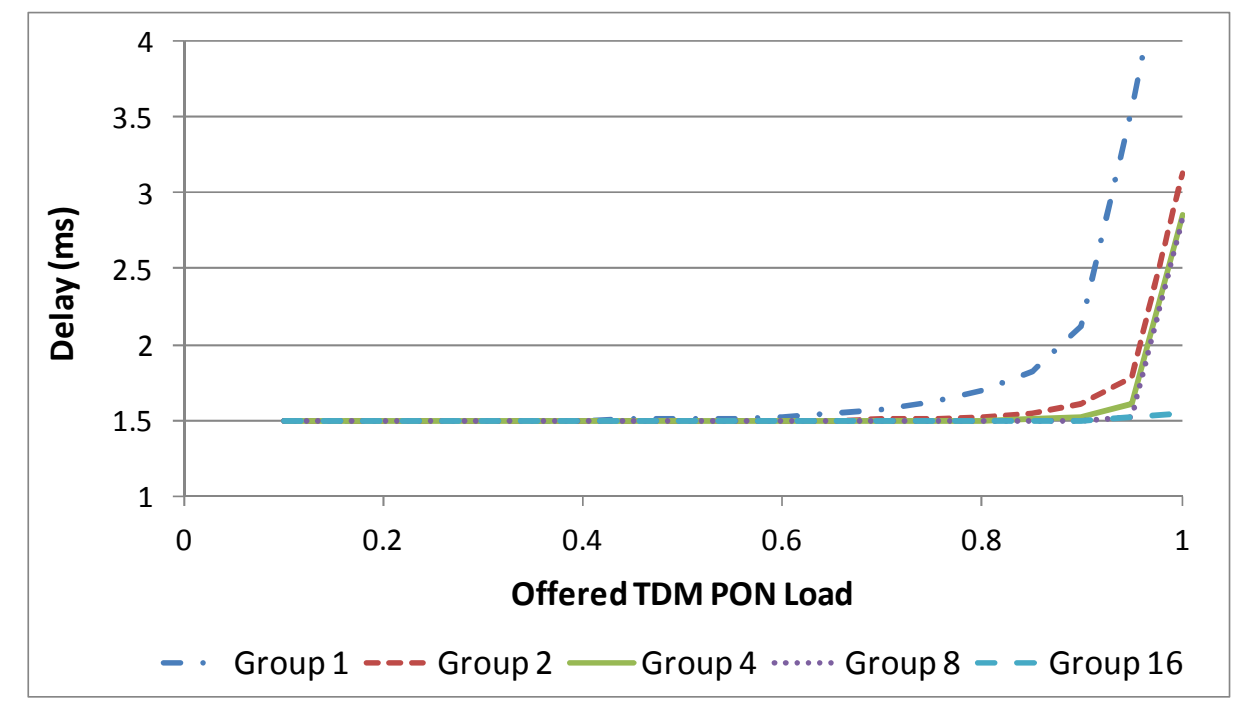

Figure 14: Average delay in function of the offered TDM PON or RN2 load, for five hybrid WDM/TDM PON variants with a different degree of flexibility in RN1 
The different simulation results clearly show that flexibility beyond a certain point does not provide much benefit. For the wavelength usage in the example scenario, this turns out to be group4. Therefore, a partially flexible architecture for RN1 is adequate enough to exploit the different advantages offered by flexibility, as listed in Section 3. Already from the moment a certain degree of flexibility is available, large gains in terms of wavelength usage are possible, but from a given point the extra gain is very limited. This outcome helps the network operator and service provider for designing a longer reach PON with a higher degree of node consolidation, as the architecture with a partially flexible RN1 provides a much lower insertion loss compared to its fully flexible counterpart.

Group2 already gives some advantage for the wavelength usage (and already great advantages for the delay), but it is clear that going to group4 definitely makes sense to increase the flexibility, at the cost of a higher insertion loss. Increasing the flexibility of group2 without largely affecting the reach, can be done by replacing the static AWG by a reconfigurable $1 \times 8$ WSS (leading to variant 1 of the B\&S-PON architecture combined with WSS, as presented in Figure 5(b)). A further flexibility increase can probably be delivered by changing the order of the WSS and power splitter (as done in Figure 5(c)). Both WSS-based solutions, however, have one or more expensive WSSs. For future work, it is important to further investigate if a WSS-based architecture with $m_{W S S}=8$ and $m_{s}=2$, is experiencing a significant flexibility increase, e.g. comparable to group4 (Figure $10(d))$.

\section{Evaluation of flexibility constraints}

As mentioned in Section 3, introducing extra flexibility in the architecture will lead to some architectural constraints, of which cost and insertion loss are the most critical ones. For the cost evaluation, a first indication of the CapEx is given for the proposed hybrid WDM/TDM architectures. A detailed OpEx calculation is out of scope for this work, but introducing some flexibility will reduce the OpEx for network extensions, energy consumption and network migration. The insertion loss calculations are combined with a reach calculation as this is the most important outcome. 
For the evaluations below, we have assumed three scenarios with $W_{u, d}=32$ wavelength pairs at $10 \mathrm{Gbps}$, and varying values for the RN1 split $M$ and the RN2 split $N$. The chosen values for $M$ and $N$, together with the resulting number of subscribers per OLT and the sustainable bandwidth per subscriber are summarized in Table 1.

Table 1: Considered scenarios for the equipment cost and insertion loss evaluation

\begin{tabular}{c||c|c|c|c} 
& M & N & $\begin{array}{c}\text { \# subscribers } \\
\text { per OLT }\end{array}$ & $\begin{array}{c}\text { Sustainable } \\
\text { bandwidth }\end{array}$ \\
\hline \hline Scenario 1 & 16 & 32 & 512 & $625 \mathrm{Mbps}$ \\
Scenario 2 & 32 & 16 & 512 & $625 \mathrm{Mbps}$ \\
Scenario 3 & 32 & 32 & 1024 & $312.5 \mathrm{Mbps}$
\end{tabular}

As an addition to Table 1, we want to make two extra remarks. First of all, in Section 2.3.2, it was mentioned that partially flexible architectures without multicasting are only of importance if $W>M$, and this means that only scenario 1 is useful for them (scenario 2 and 3 , with $W=M$, are also added in the evaluation results of these extended WS-PONs, but they are much less relevant as their functionality is almost identical to a pure WS-PON, but at a higher cost). Secondly, for the partially flexible architectures with multicasting, there is an extra design parameter in RN1 as the 1:M split is done in two different stages (see Figure 5). Here, we assume that the splitting ratio of the first stage (resp. $m_{A W G}$, $\left.m_{W S S}, m_{s}\right)$ is always equal to 4 .

\subsection{Cost}

In this section, two equipment cost evaluations are made. A first evaluation compares the equipment cost between the eight different hybrid WDM/TDM PON architectures discussed in Section 2 for the three scenarios presented in Table 1. In a second step, a more detailed evaluation is made for the different partially flexible hybrid WDM/TDM PON architectural options.

The eight architectures considered for the first evaluation are B\&S-PON, WRPON (i.e. fully flexible, cf. Figure 3), WS-PON (i.e. fully static, cf. Figure 4), B\&S-PON, combined with AWG or WSS (i.e. partially flexible with multicasting, cf. Figure 5) and WS-PON, combined with extra wavelength combiners or WSS (i.e. partially flexible without multicasting, cf. Figure 6). Table 2 gives an overview of the assumed cost figures of the most important components used in 
remote node of the different hybrid WDM/TDM PON architectures, and leading to the cost differentiation of the different architectures. Note that we assume a cost of $4 €$ per port of a passive power splitter (leading to a cost of $12 €$ for a $1: 2$ splitter, $20 €$ for a 1:4 splitter, etc.). In addition, we assume an OLT cost of ca. $200 €$ per customer if 32 users are shared per wavelength, and $400 €$ if only 16 users are shared per wavelength. Finally, for the ONU we assume a cost of $500 €$. All these costs correspond to targeted costs for within 5 years, when the first hybrid WDM/TDM PONs will probably enter the market.

Table 2: Overview of costs of the optical components used in the remote nodes of the considered hybrid WDM/TDM PONs

\begin{tabular}{l|l||l|l}
\multicolumn{1}{c|}{ Component } & \multicolumn{1}{|c|}{ Cost } & \multicolumn{1}{c}{ Component } & \multicolumn{1}{c}{ Cost } \\
\hline \hline Power splitter & $4 €($ per port) & $1 \times 2 \mathrm{WSS}$ & $4500 €$ \\
AWG & $500 €$ & $1 \times 4 \mathrm{WSS}$ & $7500 €$ \\
& & $1 \times 8 \mathrm{WSS}$ & $12500 €$
\end{tabular}

In Figure 15, the cost per subscriber is depicted for the three scenarios and the eight mentioned architectures, and a split is made between the cost for the ONU, the remote nodes (both RN1 and RN2) and the OLT. For this cost evaluation, reasonable target costs (within five years) are taken into account for the optical components, assuming a $10 \mathrm{G}$ technology (explaining the relatively high ONU costs). Note that we ignore the cost for fiber installation as this cost is identical for each architecture within a certain scenario. A full cost analysis, including fiber installation, for some hybrid WDM/TDM PON architectures is given in [21].

When evaluating the cost per subscriber for each of the proposed architectures, the main conclusion is that due to the sharing of RN1 among multiple users (i.e. 512 and 1024 in the examples of Figure 15), the cost for reconfigurability does minimally alter the overall cost per subscriber in most of the described scenarios. This opens good perspectives for advanced flexible hybrid WDM/TDM PON technologies that will probably lower the operational costs. We also clearly notice that the larger sharing ratio (1024 subscribers) for scenario 3 results in a lower cost per subscriber. However, for a few architectures, it is important to be careful in the designing phase, especially for the WR-PON and variant 2 of the B\&SPON with WSS. These are the most costly architectures, especially for scenario 2 with a large amount (i.e. 32) of small TDM PONs (i.e. 1:16 split ratio). The WR- 
PON requires a huge amount of optical switches (equal to $W_{d} \times M$ ), and the considered B\&S-PON with WSS needs a lot of WSSs (equal to twice the power split ratio $m_{s}$ at $\left.\mathrm{RN} 1\right)$.

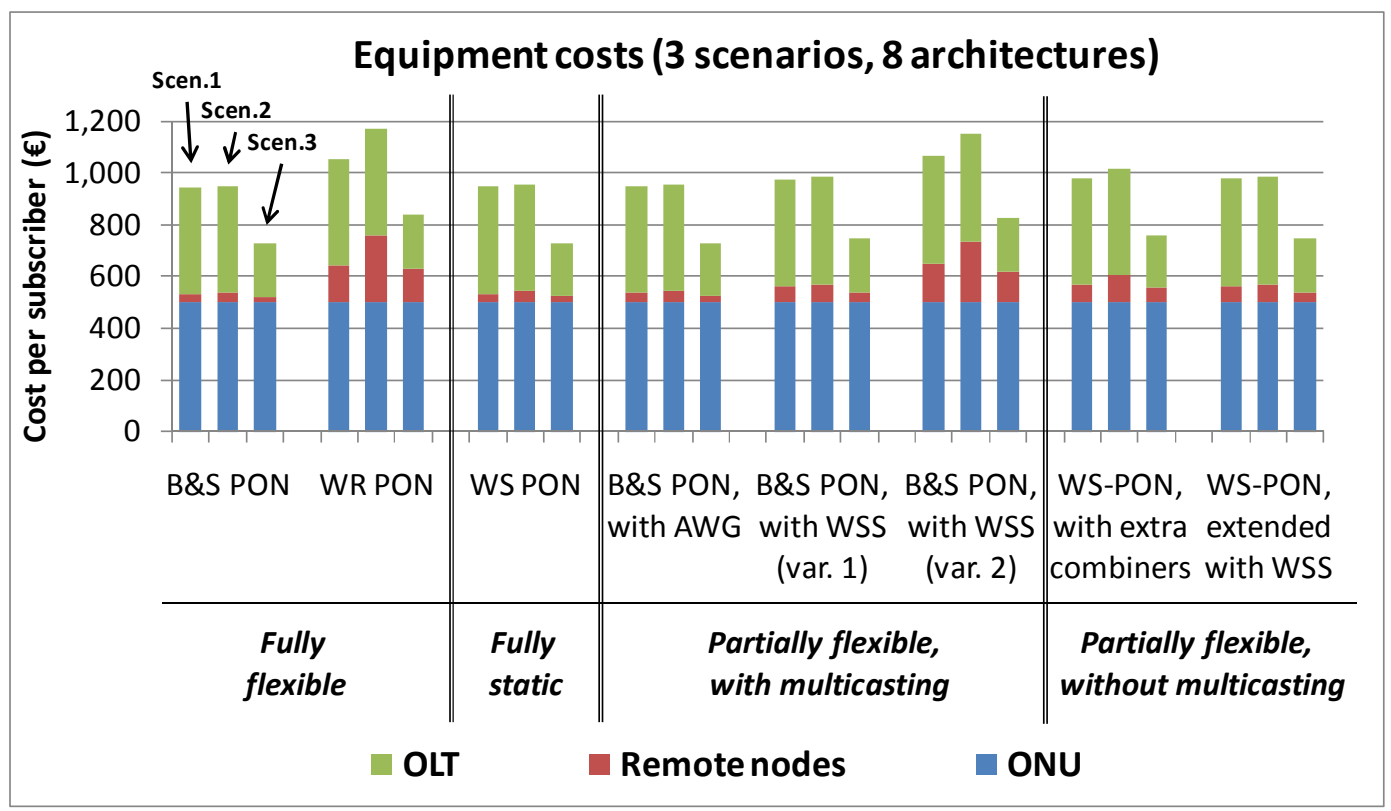

Figure 15: Cost breakdown for different WDM/TDM PON architectures

In a second evaluation, we have assumed one scenario with $W_{u, d}=16$ wavelength pairs at $10 \mathrm{Gbps}$, and an RN1 split $M$ of 1:16 and an RN2 split $N$ of 1:32. Figure 16 shows the remote nodes costs for the different variants of a partially flexible architecture with multicasting (cf. Figure 5), together with a benchmark to a B\&SPON and a WS-PON. While in Figure 15, the first splitting ratio in RN1 was equal to 4 , we have now also varied these ratios to introduce different degrees of flexibility. In each category of Figure 16, the architectures are ordered from more flexible (left, $m_{s}=8$ ) to less flexible (right, $m_{s}=2$ ).

For the B\&S-PON with AWG, the different categories exactly correspond to the architectures depicted in Figure 10. It is clear that their cost is almost independent of the flexibility. Choosing the one or the other option will mainly depend on the needed flexibility, in combination with the minimum required reach which is determined by the total insertion loss. For the B\&S-PONs in combination with WSS, we have two main variants, of which variant 2 is least interesting from a cost perspective, as already indicated in Figure 15. In function of the flexibility, we see two different cost trends for both architectural variants. In variant 1 , the cost is increasing with a decreasing flexibility (and a decreasing insertion loss), 
due to the use of WSS with a higher (up to $1 \times 8$ ) switching capability. In variant 2 , the cost is increasing with an increasing flexibility, due to the need for a higher number of WSSs (equal to $m_{s}$ ).

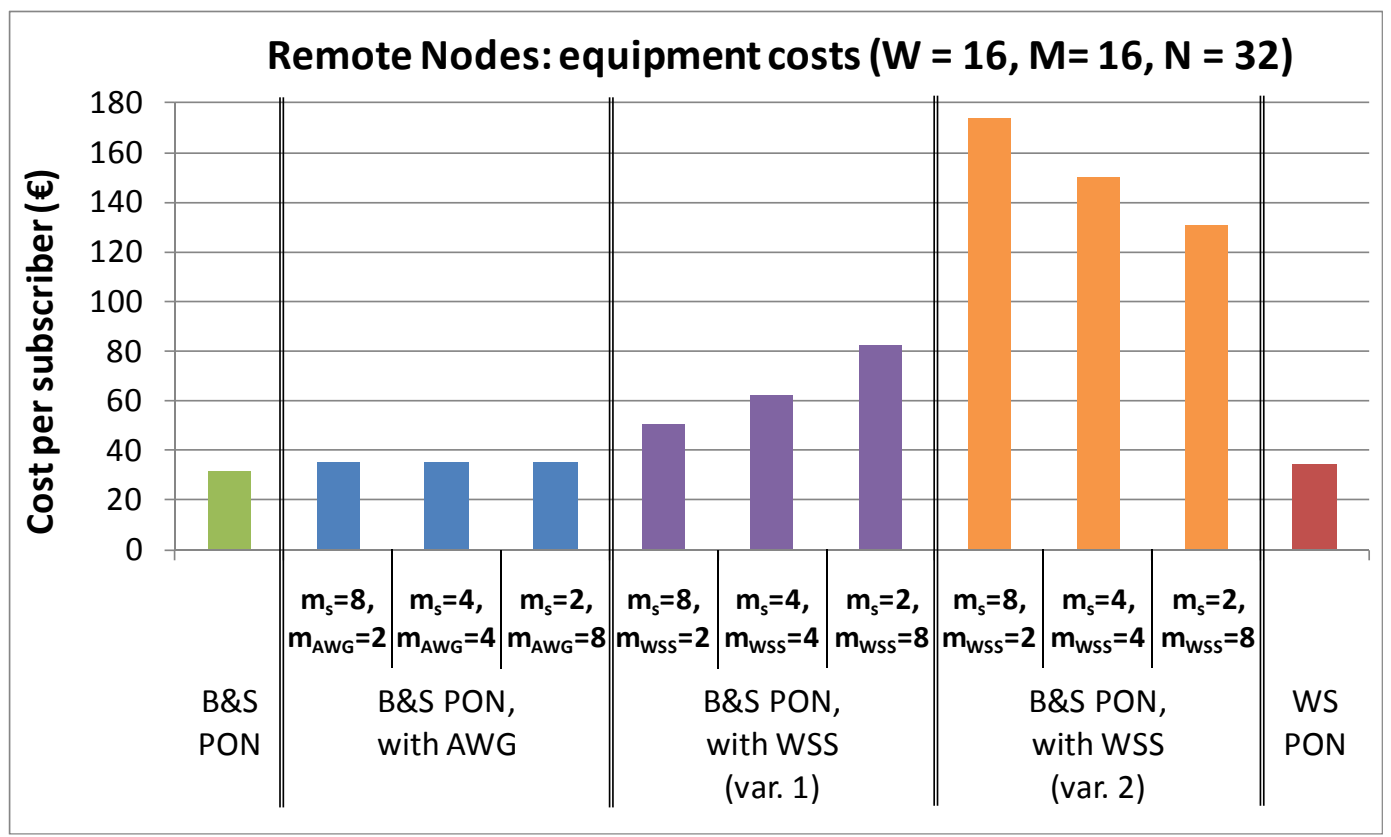

Figure 16: Remote nodes costs for different partially flexible hybrid WDM/TDM PON architectures, with multicasting (and compared to B\&S-PON and WS-PON).

\subsection{Insertion loss and reach}

In this section we calculate the insertion loss and the optical reach possible for the eight considered hybrid WDM/TDM PON architectures. Table 3 gives an overview of the assumed insertion losses of the most important components used in the different hybrid WDM/TDM PON architectures. Note that we assume a $3.5 \mathrm{~dB}$ loss per 1:2 power split instead of the theoretical loss of $3 \mathrm{~dB}$, as the latter is never attained in real implementations. For the AWG we assume a channel spacing of $100 \mathrm{GHz}$ which is sufficient for a maximum of 32 wavelengths per direction. For AWGs with a $50 \mathrm{GHz}$ spacing, however, the insertion loss will increase with roughly $1 \mathrm{~dB}$. Further, an optical component can be coupled by using a connector (ca. $0.5 \mathrm{~dB}$ ) or a splice (ca. $0.1 \mathrm{~dB}$ ). In our calculations, we use an average connector loss of $0.25 \mathrm{~dB}$. 
Table 3: Overview of insertion losses of the optical components used in the considered hybrid WDM/TDM PONs

\begin{tabular}{l|l||l|l}
\multicolumn{1}{c|}{ Component } & Insertion loss (dB) & Component & Insertion loss (dB) \\
\hline \hline 1:2-splitter & 3.5 & Connector/splice & 0.25 \\
AWG & 3 & 3-port circulator & 0.25 \\
WSS & 4 & Optical filter & 2.5
\end{tabular}

Table 4 summarizes the total insertion loss due to the optical components and the maximum optical reach possible, for the scenarios as shown in Table 1. We assume a Tx power of $3 \mathrm{dBm}$, a receiver sensitivity of $-27 \mathrm{dBm}$ (ordinary PD) and a power penalty of $2 \mathrm{~dB}$, and to calculate the optical reach, we assume a fiber loss of $0.3 \mathrm{~dB} / \mathrm{km}$. To support a longer reach, we also include an erbium-doped fiber amplifier (EDFA) in RN1 (e.g., an amplifier stage in the street cabinet), with a gain of $25 \mathrm{~dB}$ in both the uplink and downlink direction. Note that the insertion loss in the downlink direction is at least $3 \mathrm{~dB}$ higher than in the uplink direction, due to the optical filter in the ONU.

From Table 4, it is clear that a WS-PON is the best option from a reach perspective, but it has no flexibility in terms of wavelength switching. On the contrary a WR-PON (based on optical switches, combined with power splitters) is very bad in terms of optical reach: 1024 subscribers cannot even be reached with the current assumptions, and this also means that scenario 3 on Figure 15 does not make sense for the WR-PON. The main advantage of this architecture is its high security, while keeping full flexibility. However, in Section 4, it was shown that most of the time full flexibility is not required, and only a partially flexible architecture can already be of great advantage. In this way, the partially flexible architectures come into the picture. If multicasting is introduced, the reach is somewhat lowered, but in a very limited way. These B\&S-PON architectures with AWG and WSS will probably be of great importance for designing NGOA architectures, as they have a satisfactory optical reach and a reasonable amount of flexibility. Only variant 2 of the B\&S-PON with WSS will probably be too costly (as indicated in Figure 15) for arguing in favor of this architecture. 
Table 4: Insertion loss and reach of different hybrid WDM/TDM PONs

\begin{tabular}{|c|c|c|c|c|}
\hline $\begin{array}{l}\text { PON architecture } \\
\text { (upstream, } \\
\text { downstream) }\end{array}$ & $\begin{array}{c}\text { Insertion loss } \\
\text { (dB), excl. fiber } \\
\text { loss, scenario 3, } \\
1024 \text { users } \\
\end{array}$ & \begin{tabular}{|c|} 
Reach \\
$(\mathbf{k m})$, \\
scenario 1, \\
512 users \\
\end{tabular} & $\begin{array}{c}\text { Reach } \\
(\mathbf{k m}), \\
\text { scenario 2, } \\
512 \text { users } \\
\end{array}$ & $\begin{array}{c}\text { Reach } \\
(\mathbf{k m}), \\
\text { scenario 3, } \\
\text { 1024 users }\end{array}$ \\
\hline $\begin{array}{l}\text { B\&S-PON - up } \\
\text { B\&S-PON - down }\end{array}$ & $\begin{array}{l}16.5 \\
19.5 \\
\end{array}$ & 40 & 40 & 28 \\
\hline $\begin{array}{l}\text { WR-PON - up } \\
\text { WR-PON - down }\end{array}$ & $\begin{array}{l}18 \\
31\end{array}$ & 2 & 2 & I \\
\hline $\begin{array}{l}\text { WS-PON - up } \\
\text { WS-PON - down }\end{array}$ & $\begin{array}{l}3.5 \\
6.5\end{array}$ & 72 & 83 & 72 \\
\hline $\begin{array}{l}\text { B\&S-PON, AWG - up } \\
\text { B\&S-PON, AWG - down }\end{array}$ & $\begin{array}{l}14.5 \\
17.5 \\
\end{array}$ & 47 & 47 & 35 \\
\hline $\begin{array}{l}\text { B\&S-PON, WSS (1)- up } \\
\text { B\&S-PON, WSS (1) - down }\end{array}$ & $\begin{array}{l}15.5 \\
18.5\end{array}$ & 43 & 43 & 32 \\
\hline $\begin{array}{l}\text { B\&S-PON, WSS (2) - up } \\
\text { B\&S-PON, WSS (2) - down }\end{array}$ & $\begin{array}{l}12 \\
15\end{array}$ & 43 & 55 & 43 \\
\hline $\begin{array}{l}\text { WS-PON, AWG - up } \\
\text { WS-PON, AWG - down }\end{array}$ & $\begin{array}{c}7 \\
10\end{array}$ & 60 & 72 & 60 \\
\hline $\begin{array}{l}\text { WS-PON, WSS - up } \\
\text { WS-PON, WSS - down }\end{array}$ & $\begin{array}{c}8 \\
11\end{array}$ & 57 & 68 & 57 \\
\hline
\end{tabular}

\section{Conclusion}

Different hybrid WDM/TDM PON variants are discussed in this paper, and the main differences between them are highlighted. Special attention is given to flexible PON architectures, and we listed several flexibility advantages, like network performance, network extensibility, energy efficiency and network migration. Also the constraints raised by an increasing flexibility are treated, like cost, insertion loss and security issues. It is clear that there does not exist a onefits-all solution that can offer high flexibility in combination with low cost, low insertion loss and high security. However, we proved that in many cases full flexibility is not required. E.g., from the moment a certain degree of flexibility is available, large gains in terms of wavelength usage, bandwidth utilization and delay are already possible, and beyond a certain point, additional flexibility does not provide much benefit. Therefore, a partially flexible architecture is adequate enough to exploit several advantages offered by flexibility. On top of that, a cost evaluation shows that partial flexibility - by combining power splitters, arrayed waveguide gratings (AWG) and/or wavelength selective switches (WSS) - can be 
added to a hybrid WDM/TDM PON architecture without extremely affecting the final equipment cost per subscriber and probably lowering the operational cost. Moreover, using AWGs or WSSs in the first remote node of a hybrid WDM/TDM PON architecture leads to a better reach and security than for a fully flexible broadcast-and-select PON architecture only using power splitters. Therefore, considering all the selection criteria like flexibility, cost, reach and data security, a well-designed partially flexible hybrid WDM/TDM PON based on AWGs and/or WSSs provides important advantages. This opens good perspectives for offering dynamic capacity allocation in novel (partially) flexible hybrid WDM/TDM PON architectures.

Several discussed flavors in this paper, however, are long-term solutions that still need further research in a laboratory environment before they can be considered for commercial use. Moreover, the long-term flexibility advantages, like network extensibility and network migration, should be studied in more detail to quantify their importance in terms of improved network performance as well as reduced operational costs.

\section{Acknowledgements}

The research leading to these results has received funding from the European Community's Seventh Framework Programme (FP7/2007-2013) under grant agreements $n^{\circ} 212352$ (ICT-ALPHA) and nº 249025 (ICT-OASE).

\section{References}

[1] IEEE 802.3ah task force home page [Online]. Available: http://www.ieee802.org/3/efm.

[2] ITU-T G.984.x series of recommendations [Online]. Available: http://www.itu.int/rec/T-RECG/e.

[3] T. Koonen, "Fibre to the Home/Fibre to the Premises: what, where, and when?", Proc. of the IEEE, Vol. 94, No. 5, May 2006, pp. 911-934.

[4] 249025-ICT OASE Project, Requirements for European Next-Generation Optical Access Networks, D2.1.

[5] H. Rohde, S. Smolorz, and E. Gottwald, "Next Generation Ultra High Capacity PONs", 23rd Annual Meeting of the IEEE Photonics Society, Denver, Colorado, USA, Nov. 7-11, 2010.

[6] N.C. Tran, H.D. Jung, C. Okonkwo, E. Tangdiongga, and T. Koonen, “ARON: A SOA Arraybased WDM-TDM Reconfigurable Optical Access Network”, Future Network \& Mobile Summit 2010, Florence, Italy, Jun. 16-18, 2010. 
[7] H.D. Jung, N.C. Tran, E. Tangdiongga, and T. Koonen, "New Architecture for reconfigurable WDM-PON Networks based on SOA Gating Array", 9th International Conference on Optical Internet (COIN), Korea, Jul. 11-14, 2010.

[8] G. Das, B. Lannoo, H.-D. Jung, T. Koonen, D. Colle, M. Pickavet, and P. Demeester, “A New Architecture and MAC Protocol for Fully Flexible Hybrid WDM/TDM PON”, ECOC 2009, Vienna, Austria, Sep. 20-24, 2009, paper P6.28.

[9] C. Bock, J. Prat, and S. D. Walker, "Hybrid WDM/TDM PON Using the AWG FSR and Featuring Centralized Light Generation and Dynamic Bandwidth Allocation", Journal of Lightwave Technology, vol. 23, no. 12, Dec. 2005, pp. 3981-3988.

[10] N. Calabretta, M. Presi, R. Proietti, G. Contestabile, and E. Ciaramella, “A Bidirectional WDM/TDM-PON Using DPSK Downstream Signals and a Narrowband AWG”, IEEE Photonics Technology Letters, Vol. 19, No. 16, Aug. 2007, pp. 1227-1229.

[11] R. Jensen, and A. Lord, "Novel Non-Blocking Low Loss Scalable WSS Architecture", OFC/NFOEC 2008, San Diego, California, USA, Feb. 24-28, 2008, paper OthA6.

[12] G. Das, B. Lannoo, D. Colle, M. Pickavet, and P. Demeester, “A Hybrid WDM/TDM PON Architecture Using Wavelength Selective Switches", 4th IEEE International Symposium on Advanced Networks and Telecommunication Systems (ANTS 2010), Mumbai, India, Dec. 16$18,2010$.

[13] M. P. McGarry, and M. Reisslein, "Bandwidth management for WDM EPONS,” Journal of Optical Networking, Vol. 5, No. 9, Sep. 2006, pp. 637-654.

[14] A. R. Dhaini, C. M. Assi, and A. Shami, "Dynamic wavelength and bandwidth allocation in hybrid TDM/WDM EPON networks”, Journal of Lightwave Technology, Vol. 25, No. 1, Jan. 2007, pp. 277-286.

[15] M. P. McGarry, M. Reisslein, C. J. Colbourn, M. Maier, F. Aurzada, and M. Scheutzow, "Just-in-time scheduling for multichannel EPONs", Journal of Lightwave Technology, Vol. 26, No. 10, May 2008, pp. 1204-1216.

[16] K. Kanonakis, and I. Tomkos, "Improving the efficiency of online upstream scheduling and wavelength assignment in hybrid WDM/TDMA EPON networks", IEEE Journal on selected areas in Communications, Vol. 28, No. 6, Aug. 2010, pp. 838-848.

[17] W. Willinger, M. Taqqu, R. Sherman, and D. Wilson. "Self-similarity through highvariability: statistical analysis of Ethernet LAN traffic at the source level”, Proc., ACM SIGCOMM '95, Cambridge, UK, Aug. 1995, pp. 100-113.

[18] J. Zhang, and N. Ansari, "Scheduling Hybrid WDM/TDM Passive Optical Networks with Nonzero Laser Tuning Time”, IEEE/ACM Transactions on Networking, Vol. 19, No. 4, Aug. 2011, pp 1014 - 1027.

[19]C. G. Park, D. H. Han, and K. W. Rim, "Packet Delay Analysis of Symmetric Gated Polling System for DBA Scheme in an EPON", Telecommunication Systems Journal, Vol. 30, No. 13, 2005, pp. 13-34.

[20] B. Lannoo, L. Verslegers, D. Colle, M. Pickavet, M. Gagnaire, and P. Demeester, "Analytical Model for the IPACT Dynamic Bandwidth Allocation Algorithm for EPONs", Journal of Optical Networking, Vol. 6, No. 6, Jun. 2007, pp. 677-688. 
[21]B. Lannoo, G. Das, M. De Groote, D. Colle, M. Pickavet, and P. Demeester, "Technoeconomic feasibility study of different WDM/TDM PON architectures", 12th International Conference on Transparent Optical Networks (ICTON 2010), Munich, Germany, Jun. 27-Jul. $1,2010$. 